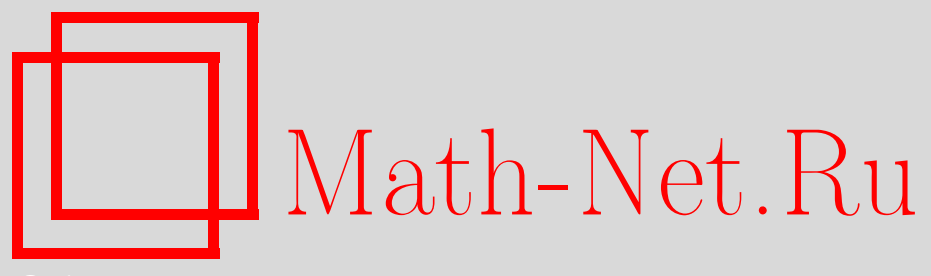

В. А. Маркашева, А. Ф. Тедеев, Задача Коши для квазилинейного параболического уравнения с градиентным стоком, Матем. сб., 2012, том 203, номер 4, 131-160

DOI: https://doi.org/10.4213/sm7744

Использование Общероссийского математического портала Math-Net.Ru подразумевает, что вы прочитали и согласны с пользовательским соглашением http://www . mathnet.ru/rus/agreement

Параметры загрузки:

IP : 54.147 .182 .235

26 апреля 2023 г., 14:39:10

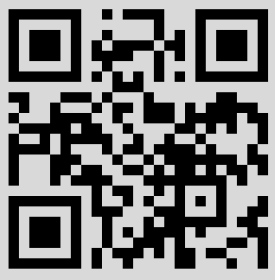




\author{
В. А. Маркашева, А. Ф. Тедеев
}

\title{
Задача Коши \\ для квазилинейного параболического уравнения с градиентным стоком
}

\begin{abstract}
В работе изучаются качественные свойства решений задачи Коши для вырождающихся параболических уравнений с нелинейным оператором типа Баоуенди-Грушина и градиентным стоком с плотностью, зависящей не только от пространственных переменных, но и от времени. Получены точные по времени оценки диаметра носителя решения и максимума решения. Найдено условие, указывающее на наличие или отсутствие эффекта убывания тотальной массы решения к нулю.

Библиография: 35 названий.
\end{abstract}

Ключевые слова: оператор типа Баоуенди-Грушина, квазилинейное параболическое уравнение, градиентный сток, убывание тотальной массы решения, оценка носителя решения.

\section{$\S 1$. Введение}

В статье исследуется решение задачи Коши для квазилинейного вырождающегося параболического уравнения следующего вида:

$$
\begin{gathered}
\frac{\partial u}{\partial t}=\operatorname{div}_{L}\left(\left|D_{L} u\right|^{\lambda-1} D_{L} u\right)-a(\rho(z)) f(t)\left|D_{L} u^{\nu}\right|^{q}, \\
(z, t) \in S_{T}=\mathbb{R}^{N+M} \times(0, T), \\
u(z, 0)=u_{0}(z) \in L_{1}\left(\mathbb{R}^{M+N}\right), \quad u_{0}(z) \geqslant 0 \quad \text { п.в. } \\
z=(x, y), \quad x \in \mathbb{R}^{N}, \quad y \in \mathbb{R}^{M} .
\end{gathered}
$$

Здесь $\lambda>1,1<q<\lambda+1, \nu q>\lambda$, а $x=\left(x_{1}, \ldots, x_{N}\right), y=\left(y_{1}, \ldots, y_{M}\right)-$ произвольные точки евклидовых пространств $\mathbb{R}^{N}$ и $\mathbb{R}^{M}$ соответственно, $N \geqslant 1$, $M \geqslant 1$. Пусть $f(t), a(\rho(z))$ - неотрицательные и измеримые функции. И кроме того,

$$
\begin{gathered}
a(s) \text { - непрерывная неубывающая функция такая, } \\
\text { что для всех } s>0 \text { функция } s^{q} / a(s) \text { также не убывает. }
\end{gathered}
$$

Функция $\rho(z)=\left(|x|^{2(\alpha+1)}+(\alpha+1)^{2}|y|^{2}\right)^{1 / 2(\alpha+1)}$ является аналогом функции расстояния между точками евклидова пространства и будет более подробно описана в $§ 2$.

$$
\begin{gathered}
f(t)-\text { непрерывная неубывающая функция такая, } \\
\text { что для всех } t>0 \text { найдется такое число } \mu, \\
0<\mu<(\nu q-\lambda) / \lambda, \text { что функция } t^{\mu} / f(t) \text { также не убывает. }
\end{gathered}
$$

(C) В. А. Маркашева, А. Ф. Тедеeb, 2012 
Символом $D_{L} u$ обозначим вектор

$$
D_{L} u=\left(\frac{\partial u}{\partial x_{1}}, \frac{\partial u}{\partial x_{2}}, \ldots, \frac{\partial u}{\partial x_{N}},|x|^{\alpha} \frac{\partial u}{\partial y_{1}},|x|^{\alpha} \frac{\partial u}{\partial y_{2}}, \ldots,|x|^{\alpha} \frac{\partial u}{\partial y_{M}}\right) .
$$

Векторное поле, соответствующее этому вектору, часто называют векторным полем Баоуенди-Грушина (см. [1], [2]). По аналогии оператор, определенный при помощи этих векторных полей, будем называть оператором типа БаоуендиГрушина. В дальнейшем считаем, что $\alpha>0$, поскольку теоремы вложения, которые мы будем существенно использовать, доказаны для этого случая. Далее,

$$
\begin{gathered}
\left|D_{L} u\right|=\sqrt{\sum_{i=1}^{N}\left(\frac{\partial u}{\partial x_{i}}\right)^{2}+|x|^{2 \alpha} \sum_{j=1}^{M}\left(\frac{\partial u}{\partial y_{j}}\right)^{2}}, \\
\operatorname{div}_{L} \vec{F}(x, y)=\sum_{i=1}^{N} \frac{\partial F_{i}}{\partial x_{i}}+|x|^{\alpha} \sum_{j=1}^{M} \frac{\partial F_{j+N}}{\partial y_{j}} .
\end{gathered}
$$

Слагаемое $-a(\rho(z)) f(t)\left|D_{L} u^{\nu}\right|^{q}$ с физической точки зрения оказывает демпфирующий эффект и в дальнейшем будет называться демпфирующим членом уравнения.

Определим действие $L_{\lambda, \alpha}[u]$ оператора типа Баоуенди-Грушина на произвольную функцию

$$
L_{\lambda, \alpha}[u]:=\operatorname{div}_{L}\left(\left|D_{L} u\right|^{\lambda-1} D_{L} u\right) .
$$

Уравнение с оператором типа Баоуенди-Грушина представляет независимый интерес. Если рассмотреть (1.1) без демпфирования и положить $\alpha=0$, уравнение описывает процесс с медленной диффузией (см. обзор [3]). Операторы типа $L_{1, \alpha}=\Delta_{x}+|x|^{2 \alpha} \Delta_{y}$, где символы $\Delta_{x}$ и $\Delta_{y}$ обозначают операторы Лапласа по переменным $x$ и $y$ соответственно, впервые исследовались в работах [4] и [5]. В работах [6] и [7] изучались качественные свойства решения уравнения $L_{\lambda, \alpha}[u]=f$, т.е. эллиптического аналога (1.1) при отсутствии демпфирования (см. также [1] и имеющуюся там литературу). В работе [8] при отсутствии демпфирующего члена были изучены вопросы существования решения задачи Коши, получены точные оценки максимума решения и скорость распространения носителя. В [9], [10] доказана локальная гёльдеровость решения.

Интерес к задаче (1.1), (1.2) вызван тем, что наличие демпфирующего члена в виде градиентной абсорбции при определенных условиях может сильно менять свойства решения. А именно: создает эффект убывания массы решения с течением времени. В случае $\alpha=0, a(s)=f(t)=1$ свойства решений уравнения (1.1) были исследованы в множестве работ, например, [11]-[17] для обычного оператора Лапласа и [18]-[22] для оператора $\lambda$-Лапласа.

В статье [23] рассматривалась задача Коши для уравнения с двойной нелинейностью и источником

$$
\begin{gathered}
\frac{\partial u}{\partial t}=\operatorname{div}\left(u^{m-1}|D u|^{\lambda-1} D u\right)-\varepsilon\left|D u^{\nu}\right|^{q}+\delta u^{p}, \\
u(x, 0)=u_{0}(x) \in L_{1}\left(\mathbb{R}^{N}\right),
\end{gathered}
$$

$(x, t) \in S_{T}=\mathbb{R}^{N} \times(0, T), T>0, p>1$, с неотрицательной начальной функцией из $L_{1}\left(\mathbb{R}^{N}\right)$ при условии $\varepsilon, \delta \geqslant 0, m+\lambda-2>0, \lambda>0,1<q<\lambda+1, \nu q>m+\lambda-1$. 
Это уравнение, как легко видеть, при $\delta=0, m=1$ является частным случаем (1.1) и совпадает с (1.1) при $\alpha=0, a(s)=\varepsilon, f(t)=1$. В этой работе было найдено критическое условие на $q$, при котором тотальная масса решения стремится к нулю. Ранее такого сорта результаты для почти линейных уравнений были установлены во многих работах, например в [11], [13], [15], [17]. Результаты, полученные при рассмотрении уравнения с демпфирующим членом, хотя и имеют самостоятельную ценность, были использованы авторами работы [23] для исследования вопроса существования решения задачи Коши для уравнения с источником и демпфированием.

Целью настоящей работы является получение условия на параметры задачи, при которых гарантируется наличие или отсутствие эффекта убывания к нулю тотальной массы решения. Более того, наши подходы позволяют нам найти скорость убывания тотальной массы. Получены оценки сверху максимума решения и радиуса носителя решения задачи Коши (1.1), (1.2). По-видимому, найденные оценки точны. Однако имеет смысл доказать аналогичные оценки снизу. Также найден предельный показатель $q *$ и найдена зависимость показателя, характеризующая порядок убывания массы решения, для специального случая нелинейного веса типа $a(s)=s^{\gamma}, f(t)=t^{\beta}$ при демпфирующем члене.

Введем теперь понятие слабого решения задачи (1.1), (1.2). Пусть выполнены условия $\left(\mathrm{H}_{1}\right),\left(\mathrm{H}_{2}\right)$.

ОПРЕДЕЛЕНиЕ 1 . Неотрицательную функцию $u(z, t) \in L_{\infty, \text { loc }}\left(S_{T}\right)$ будем называть слабым решением уравнения (1.1) в $S_{T}=\mathbb{R}^{N+M} \times(0, T)$, если для любого $t, T>t>0, R>0$,

$$
\begin{gathered}
u \in C\left((0, T), L_{2, \text { loc }}\left(\mathbb{R}^{N+M}\right)\right), \\
\left|D_{L} u\right|^{\lambda+1}, a(\rho(z)) f(t)\left|D_{L} u^{\nu}\right|^{q} \in L_{1, \mathrm{loc}}\left(S_{T}\right)
\end{gathered}
$$

и $u$ удовлетворяет (1.1) в смысле интегрального тождества

$$
\iint_{B_{R} \times(t, T)}-u \eta_{\tau}+\left(\left|D_{L} u\right|^{\lambda-1} D_{L} u\right) D_{L} \eta+\eta a(\rho(z)) f(\tau)\left|D_{L} u^{\nu}\right|^{q} d x d y d \tau=0,
$$

где $\eta(x, y, t)$ - произвольная гладкая функция с носителем из $B_{R} \times(t, T)$.

ОПРЕДЕЛЕниЕ 2. Слабое решение уравнения (1.1) $u(z, t) \in L_{\infty, \mathrm{loc}}\left(S_{T}\right)$ будем называть слабым решением задачи (1.1), (1.2), если

$$
\lim _{t \rightarrow 0} \int_{\mathbb{R}^{N+M}} u \eta(z) d z=\int_{\mathbb{R}^{N+M}} u_{0} \eta(z) d z \quad \forall \eta(z) \in C_{0}^{\infty}\left(\mathbb{R}^{N+M}\right) .
$$

Прежде чем перейти к формулировкам основных результатов работы, введем необходимые обозначения. Здесь и далее

$$
\begin{gathered}
K=Q(\lambda-1)+\lambda+1, \quad K_{1+\theta}=Q(\lambda-1)+(\lambda+1)(1+\theta), \\
H=(\lambda+1)(\nu q-1)-q(\lambda-1)>0, \quad Q=N+M(\alpha+1), \\
B_{R}=\left\{z \in \mathbb{R}^{N+M}: \rho(z, 0) \leqslant R\right\},
\end{gathered}
$$

где $\rho(z)=\left(|x|^{2(\alpha+1)}+(\alpha+1)^{2}|y|^{2}\right)^{1 / 2(\alpha+1)}$ уже было определено выше. Параметр $K$ можно сравнить с показателем Баренблатта, и при $\alpha=0$ он играет ключевую роль в описании качественных свойств решения вырождающихся уравнений (см. [3]), $Q$ - однородная размерность в пространствах Карно-Каратеодори 
(подробнее см. [1]). Наконец, $B_{R}$ является естественным расширением понятия открытого шара в пространствах Карно-Каратеодори. Более подробное описание отнесено к $\S 2$. На протяжении всей работы символами $C, C_{i}, C_{i}^{\prime}$ будем обозначать различные положительные постоянные, зависящие лишь от параметров $\lambda, N, M, \alpha, \nu, q$.

Радиусом носителя решения будем называть величину

$$
Z(t)=\inf \left\{\rho: u(\cdot, t)=0 \text { для п.в. }(x, y) \in \mathbb{R}^{N+M} \backslash B_{\rho}\right\} .
$$

Обозначим $\varphi(s)$ функцию, обратную к $a(s)^{\lambda-1} s^{H}$.

Важную роль в работе играет функция

$$
\omega(t) \equiv \frac{\varphi\left(t^{\nu q-\lambda} / f(t)^{\lambda-1}\right)}{t^{1 / K}} .
$$

Приступим к формулировке основных результатов работы. Нам потребуются следующие асимптотические условия на $\omega(t)$ :

$$
\begin{gathered}
\lim _{t \rightarrow \infty} \omega(t)=0, \\
\exists C_{1}>0: \quad C_{1} \leqslant \lim _{t \rightarrow \infty} \omega(t),
\end{gathered}
$$

для достаточно больших $t$

$$
\exists C_{2}, \epsilon>0: \quad C_{2} t^{\epsilon} \leqslant \omega(t) .
$$

На примере специального веса условие (1.8) превратится в условие на предельный показатель $q^{*}$.

ТеОрема 1. Пусть выполненъ условия $\left(\mathrm{H}_{1}\right),\left(\mathrm{H}_{2}\right)$. Тогда существует и слабое решение (1.1), (1.2), и если $\omega(t)$ - функиия, определенная в (1.6), $\operatorname{supp} u_{0} \subset B_{R_{0}}, R_{0}<\infty$, то для достаточно больших $t$ имеем

$$
Z(t) \leqslant C_{3} \omega(t) t^{\frac{1}{K}}
$$

ЗАмечАниЕ 1. Вопрос единственности решения задачи (1.1), (1.2) остается открытым. Трудность доказательства единственности связана с наличием градиентного стока в уравнении и может быть преодолена, например, с помощью равномерных оценок градиента решения, что, в свою очередь, является нетривиальной задачей.

ЗАмЕчАниЕ 2. Если выполняется (1.7), то из (1.10) следует, что при достаточно больших $t$ оценка (1.10) сильнее оценки носителя для решения того же уравнения без стока, т.е. для $a(s)=f(t)=0$ (см. предложение 7 далее или [8]).

Теорема 2. Пусть и - слабое решение (1.1), (1.2) и выполнены условия $\left(\mathrm{H}_{1}\right),\left(\mathrm{H}_{2}\right), \operatorname{supp} u_{0} \subset B_{R_{0}}, R_{0}<\infty$. И пусть $0<\alpha<M(\nu q-1) / q$. Тогда для достаточно больших $t$ имеем

$$
\begin{gathered}
\int_{\mathbb{R}^{M+N}} u d z \leqslant C_{4} \omega^{\frac{K}{\lambda-1}}(t), \\
\|u\|_{\infty, \mathbb{R}^{N+M}} \leqslant C_{5} \omega^{\frac{\lambda+1}{\lambda-1}}(t) t^{-\frac{Q}{K}} .
\end{gathered}
$$

ЗАмЕЧАнИЕ 3. В случае, если выполняется (1.7), неравенство (1.11) обеспечивает убывание к нулю тотальной массы решения $\|u\|_{1, \mathbb{R}^{N+M}}$. 
Рассмотрены также два случая, при которых можно получить оценку массы решения без предположения компактности носителя начальной функции.

Теорема 3. Пусть и - слабое решение (1.1), (1.2) и выполнены условия $\left(\mathrm{H}_{1}\right),\left(\mathrm{H}_{2}\right)$. Тогда для достаточно больших $t$ при условии, что $\nu=1, \lambda>1 u$ $0<\alpha<M(q-1) / q$, справедлива оченка

$$
\int_{\mathbb{R}^{N+M}} u(t) d z \leqslant \int_{\rho>\omega(t) t^{\frac{1}{K}}} u_{0} d z+C_{6} \omega^{\frac{K}{\lambda-1}}(t) .
$$

Если же $\lambda=1$ и $0<\alpha<M(\nu q-1) / q$, то справедлива оченка

$$
\int_{\mathbb{R}^{N+M}} u(t) d z \leqslant \int_{\rho>\sqrt{t}} u_{0} d z+C_{6}^{\prime} \frac{t^{\frac{Q(\nu q-1)+q-2}{2(\nu q-1)}}}{a(\sqrt{t})^{\frac{1}{\nu q-1}} f(t)^{\frac{1}{\nu q-1}}} .
$$

ЗАмЕчАнИЕ 4. В случае, если выполняется (1.7), неравенство (1.13) обеспечивает убывание к нулю тотальной массы решения $\|u\|_{1, \mathbb{R}^{N+M}}$.

Из оценки (1.14) следует, например, что в случае, если

$$
q<\frac{Q+2}{\nu Q+1}
$$

тотальная масса решения стремится к нулю при $t \rightarrow \infty$. Заметим также, что при $\alpha=0, \nu=1, a=f=1$ критическим показателем является $q=(M+N+2) /$ $(M+N+1)$ (см., например, [12]).

Теорема 4. Пусть и - слабое решение (1.1), (1.2) и выполнены условия $\left(\mathrm{H}_{1}\right),\left(\mathrm{H}_{2}\right), \operatorname{supp} u_{0} \subset B_{R_{0}}, R_{0}<\infty$. Если выполняется условие (1.8), тогда для достаточно больших $t, t \geqslant t_{0}=\left\|u_{0}\right\|_{1, \mathbb{R}^{N+M}}^{\lambda-1} / R_{0}^{K}$, имеем

$$
\int_{\mathbb{R}^{N+M}} u d z \leqslant C_{7}\left(\int_{t_{0}}^{t} \frac{a\left(\tau^{\frac{1}{K}}\right) f(\tau)}{\tau^{\frac{Q(\nu q-1)+q}{K}}} d \tau\right)^{-\frac{1}{\nu q-1}} .
$$

В частности, из (1.15) следует, что если существуют $C>0, \varrho \in(0,1)$ такие, что $C \varrho \leqslant \omega(t) \leqslant C$, то для достаточно больших $t, t \geqslant t_{0}=\left\|u_{0}\right\|_{1, \mathbb{R}^{N+M}}^{\lambda-1} / R_{0}^{K}$, имеем

$$
\int_{\mathbb{R}^{N+M}} u d z \leqslant C_{8}\left(\ln \left(\frac{t}{t_{0}}\right)\right)^{-\frac{1}{\nu q-1}} .
$$

Теорема 5. Пусть и - слабое решение (1.1), (1.2) и выполнены условия $\left(\mathrm{H}_{1}\right),\left(\mathrm{H}_{2}\right), \operatorname{supp} u_{0} \subset B_{R_{0}}, R_{0}<\infty$. Если выполняется условие (1.9), тогда для достаточно больших $t$ имеем

$$
\int_{\mathbb{R}^{N+M}} u(t) d z \geqslant C_{9}>0,
$$

где $C_{9}$ - положительная постоянная, зависящая только от параметров задачи $и\left\|u_{0}\right\|_{1}$.

Пример. Пусть $u$ - слабое решение (1.1), (1.2), supp $u_{0} \subset B_{R_{0}}, R_{0}<\infty$. Пусть $a(s)=s^{\gamma}, \gamma<q, f(t)=t^{\beta}, \beta<\frac{\nu q-\lambda}{\lambda}$,

$$
q^{*}=\frac{Q+\gamma+K(1+\beta)}{\nu Q+1}, \quad A=\frac{\left(q^{*}-q\right)(\nu Q+1)}{H+\gamma(\lambda-1)} .
$$


Тогда $\omega(t)=t^{-\frac{A(\lambda-1)}{K}}$. Отметим, что $q^{*}$ является критическим показателем, поскольку если $q<q^{*}$, тогда выполняется условие (1.7), оценки (1.10), (1.11), (1.12) справедливы и имеют вид

$$
\begin{gathered}
Z(t) \leqslant C_{10} t^{\frac{1}{K}-\frac{A(\lambda-1)}{K}}, \\
\int_{\mathbb{R}^{N+M}} u(t) d z \leqslant C_{11} t^{-A}, \\
\|u(t)\|_{\infty, \mathbb{R}^{N+M}} \leqslant C_{12} t^{-\frac{Q}{K}-\frac{A(\lambda+1)}{K}} .
\end{gathered}
$$

Следует добавить, что неравенство (1.8) превращается в равенство в случае, когда $q=q^{*}$, и оценка (1.16) остается неизменной. Таким образом, эффект убывания массы решения к нулю сохраняется при $q \leqslant q^{*}$.

В случае, когда $q^{*}<q<\lambda+1$, выполняется условие (1.9), масса убывает, но ограничена снизу положительной константой, как показывает оценка теоремы 5 .

Найденный критический показатель совпадает с полученными в работах [23] при условии $\alpha=0, a(s) f(t) \equiv \varepsilon>0$, и [24] при условии $\alpha=0, f(t) \equiv 1$.

Параграф 2 посвящен вспомогательным предложениям, которые приведены без доказательства. Параграфы 3, 4, 5, 6, 7 посвящены доказательству теорем $1,2,3,4,5$ соответственно.

В доказательстве теорем 1-5 мы используем подходы работ [23], [24] и [8].

В доказательстве оценок размеров носителя решения мы использовали идеи работ [25]-[27], а также существенно опирались на работы [23], [24] и [8], где эти методы получили дальнейшее развитие.

\section{§ 2. Вспомогательные предложения}

Символом $D_{L} u$ выше обозначен вектор

$$
D_{L} u=\left(\frac{\partial u}{\partial x_{1}}, \frac{\partial u}{\partial x_{2}}, \ldots, \frac{\partial u}{\partial x_{N}},|x|^{\alpha} \frac{\partial u}{\partial y_{1}},|x|^{\alpha} \frac{\partial u}{\partial y_{2}}, \ldots,|x|^{\alpha} \frac{\partial u}{\partial y_{M}}\right) .
$$

Векторное поле, образующее этот вектор, называется векторным полем Баоуенди-Грушина. Введем вспомогательные обозначения:

$$
\begin{gathered}
\frac{\partial u}{\partial x_{1}}=X_{1}, \quad \frac{\partial u}{\partial x_{2}}=X_{2}, \quad \ldots, \quad \frac{\partial u}{\partial x_{N}}=X_{N}, \\
|x|^{\alpha} \frac{\partial u}{\partial y_{1}}=Y_{1}, \quad|x|^{\alpha} \frac{\partial u}{\partial y_{2}}=Y_{2}, \quad \ldots, \quad|x|^{\alpha} \frac{\partial u}{\partial y_{M}}=Y_{M} .
\end{gathered}
$$

Известно, что если $\alpha$ - четное положительное целое число, то $C^{\infty}$-векторные поля $X_{1}, X_{2}, \ldots, X_{N}, Y_{1}, \ldots, Y_{M}$ удовлетворяют условию ранга Хёрмандера

$$
\operatorname{rank} \operatorname{Lie}\left[X_{1}, X_{2}, \ldots, X_{N}, Y_{1}, \ldots, Y_{M}\right]=M+N .
$$

Для данного набора $C^{\infty}$-векторных полей определяется метрическое пространство Карно-Каратеодори (см. [1], [28]-[30]), где расстояние можно ввести по формуле $\rho\left(z, z^{0}\right)=\left(\left|x-x^{0}\right|^{2(\alpha+1)}+(\alpha+1)^{2}\left|y-y^{0}\right|^{2}\right)^{1 / 2(\alpha+1)}, z=(x, y), z^{0}=$ 
$\left(x^{0}, y^{0}\right)$. Действительно, введенная функция удовлетворяет стандартным требованиям однородности и симметричности. Неравенство треугольника также выполняется, но с константой $C$ больше единицы, т.е.

$$
\forall z^{1}, z^{2}, z^{3} \in \mathbb{R}^{N+M} \quad \rho\left(z^{1}, z^{2}\right) \leqslant C\left(\rho\left(z^{1}, z^{3}\right)+\rho\left(z^{3}, z^{2}\right)\right) .
$$

Определим анизотропное растяжение, применимое к $L_{\lambda, \alpha}$, следующим образом:

$$
\delta_{\mu}(z)=\left(\mu x, \mu^{\alpha+1} y\right), \quad \mu>0, \quad z=(x, y) \in \mathbb{R}^{N+M} .
$$

Введенное расстояние называется однородным, поскольку для всех $z \in \mathbb{R}^{N+M}$ выполняется тождество

$$
\rho\left(\delta_{\mu}(z)\right)=\mu \rho(z) .
$$

Очевидно, что замена переменных в интеграле Лебега дает

$$
d \circ \delta_{\mu}(x, y)=\mu^{Q} d x d y, \quad Q=N+(\alpha+1) M,
$$

где $Q$ есть однородная размерность в пространствах Карно-Каратеодори.

Пусть $\Omega \subset \mathbb{R}^{N+M}$ - произвольное ограниченное открытое множество в $R^{N+M}$ с достаточно гладкой границей. Определим пространство $\mathfrak{L}_{1, p}(\Omega)$ для $1 \leqslant$ $p<\infty$ как замыкание $C^{\infty}(\bar{\Omega})$ по норме

$$
\|f\|_{\mathfrak{L}^{1, p}(\Omega)}=\left(\int_{\Omega}\left(\left|D_{L} f\right|^{p}+|f|^{p}\right) d x d y\right)^{\frac{1}{p}},
$$

которая эквивалентна норме

$$
\left(\int_{\Omega}\left|D_{L} f\right|^{p} d x d y\right)^{\frac{1}{p}}+\left(\int_{\Omega}|f|^{r} d x d y\right)^{\frac{1}{r}}
$$

для $0<r \leqslant p$.

Обозначим через $\stackrel{\circ}{\mathfrak{L}}_{1, p}(\Omega)$ подпространство $\mathfrak{L}_{1, p}(\Omega)$, которое получается как замыкание $C_{0}^{\infty}(\Omega)$ по норме $\mathfrak{L}_{1, p}(\Omega)$. Пространства $\mathfrak{L}_{1, p}(\Omega)$ и $\stackrel{\circ}{\mathfrak{L}}_{1, p}(\Omega)$ включаются в класс пространств Карно-Каратеодори, для которых достаточно полно изучены теоремы вложения типа С. Л. Соболева и мультипликативные неравенства типа Гальярдо-Ниренберга. Читателя, интересующегося теорией пространств Карно-Каратеодори, мы отсылаем к обзорной работе [1], где можно найти дальнейшую литературу.

ПредложениЕ 1 (неравенство типа Гальярдо-Ниренберга; см. [1]). Для любой функиии $f \in \mathfrak{L}_{1, \lambda+1}\left(\mathbb{R}^{N+M}\right)$ справедливо неравенство

$$
\int_{\mathbb{R}^{N+M}}|f|^{\lambda+1} d z \leqslant C_{14}\left(\int_{\mathbb{R}^{N+M}}\left|D_{L} f\right|^{\lambda+1} d z\right)^{\beta_{1}}\left(\int_{\mathbb{R}^{N+M}}|f|^{\beta_{2}} d z\right)^{\frac{\left(1-\beta_{1}\right)(\lambda+1)}{\beta_{2}}} .
$$

Здесъ $\beta_{1}=\frac{Q(\lambda-1)}{K_{1+\theta}}, \beta_{2}>0$.

ПредложениЕ 2 (неравенство типа Пуанкаре; см. [1]). Для любой функиии $\omega \in \stackrel{\circ}{\mathfrak{L}}_{1, q}\left(\mathbb{R}^{N+M}\right)$ справедливо неравенство

$$
\int_{\Omega}|\omega|^{q} d z \leqslant C_{15} \operatorname{mes}\{\operatorname{supp} \omega\}^{\frac{q}{Q}} \int_{\Omega}\left|D_{L} \omega\right|^{q} d z .
$$


Следующее предложение не следует из работы [2], но его легко доказать аналогичными методами.

ПредложениЕ 3 (неравенство типа Харди). Пусть $а(s)$ - непрерьвная монотонно возрастающая функция такая, что $s^{q} / a(s)$ также монотонно возрастает. Тогда для любой функиии $\omega \in \stackrel{\circ}{\mathfrak{L}}_{1, q}(\Omega)$ справедливо неравенство

$$
\int_{\Omega} a(\rho(z)) \frac{\left|D_{L} \rho\right|^{q}}{\rho^{q}}|\omega|^{q} d z \leqslant\left(\frac{q}{Q}\right)^{q} \int_{\Omega} a(\rho)\left|D_{L} \omega\right|^{q} d z .
$$

Для любой функиии $\omega \in \mathfrak{L}_{1, q}\left(\mathbb{R}^{N+M}\right)$ справедливо неравенство

$$
\int_{\mathbb{R}^{N+M}} a(\rho(z)) \frac{\left|D_{L} \rho\right|^{q}}{\rho^{q}}|\omega|^{q} d z \leqslant\left(\frac{q}{Q}\right)^{q} \int_{\mathbb{R}^{N+M}} a(\rho)\left|D_{L} \omega\right|^{q} d z .
$$

ПредложениЕ 4 (о замене переменных). Пусть

$$
\rho(z, 0)=\left(|x|^{2(\alpha+1)}+(\alpha+1)^{2}|y|^{2}\right)^{\frac{1}{2(\alpha+1)}} .
$$

Следуя формулам

$$
\left\{\begin{array}{l}
x_{1}=\rho \cos ^{\frac{1}{\alpha+1}} \varphi_{M+N-1} \cos ^{\frac{1}{\alpha+1}} \varphi_{M+N-2} \cos ^{\frac{1}{\alpha+1}} \varphi_{2} \cos ^{\frac{1}{\alpha+1}} \varphi_{1}, \\
x_{2}=\rho \cos ^{\frac{1}{\alpha+1}} \varphi_{M+N-1} \cos ^{\frac{1}{\alpha+1}} \varphi_{M+N-2} \cos ^{\frac{1}{\alpha+1}} \varphi_{2} \sin ^{\frac{1}{\alpha+1}} \varphi_{1}, \\
x_{M}=\rho \cos ^{\frac{1}{\alpha+1}} \varphi_{M+N-1} \cos ^{\frac{1}{\alpha+1}} \varphi_{M+N-2} \cos ^{\frac{1}{\alpha+1}} \varphi_{M} \sin ^{\frac{1}{\alpha+1}} \varphi_{M-1}, \\
y_{1}=\frac{\rho^{\alpha+1}}{\alpha+1} \cos \varphi_{M+N-1} \cos \varphi_{M+N-2} \cos \varphi_{M+1} \sin \varphi_{M}, \\
y_{2}=\frac{\rho^{\alpha+1}}{\alpha+1} \cos \varphi_{M+N-1} \cos \varphi_{M+N-2} \cos \varphi_{M+2} \sin \varphi_{M+1}, \\
\ldots \\
y_{N}=\frac{\rho^{\alpha+1}}{\alpha+1} \sin \varphi_{M+N-1},
\end{array}\right.
$$

заменим координаты $(x, y)$ на $(\rho, \varphi)$. Якобиан замены равен

$$
\begin{aligned}
& J(\rho, \varphi)=\frac{\partial(x, y)}{\partial(\rho, \varphi)}=\frac{\cos ^{\frac{1}{\alpha+1}} \varphi_{2} \cos ^{\frac{2}{\alpha+1}} \varphi_{3} \cdots \cos ^{\frac{M-2}{\alpha+1}} \varphi_{M-1} \cos ^{\frac{M-1}{\alpha+1}} \varphi_{M} \cos ^{\frac{M}{\alpha+1}} \varphi_{M+1}}{\left(\cos \varphi_{1} \cdots \cos \varphi_{M}\right)^{\frac{\alpha}{\alpha+1}}} \\
& \times \frac{\cos ^{\frac{M}{\alpha+1}+1} \varphi_{M+2} \cdots \cos \frac{M}{\alpha+1}+N-2}{\varphi_{M+N-1}} .
\end{aligned}
$$

В дальнейшем нам потребуется итерационная лемма.

ПредложениЕ 5 (см. [31; лемма 5.6] при $b>1$ ). Пусть последовательность $y_{h}, h=0,1,2, \ldots$, неотрицательных чисел удовлетворяет рекуррентному соотношению

$$
y_{h+1} \leqslant C_{16} b^{h} y_{h}^{1+\varepsilon}, \quad h=0,1,2, \ldots,
$$

с какими-либо положительными постоянными $C_{16}, \varepsilon>0$ u $b>1$. Тогда, если

$$
y_{0} \leqslant C_{16}^{-\frac{1}{\varepsilon}} b^{-\frac{1}{\varepsilon^{2}}}, \quad \text { mo } y_{h} \rightarrow 0 \quad \text { npu } h \rightarrow \infty .
$$


ПРЕДЛОЖЕНИЕ 6 (см. [32; гл. I, лемма 4.3]). Пусть последовательность $y_{h}$, $h=0,1,2, \ldots$, неотрищательных чисел удовлетворяет рекуррентному соотношению

$$
y_{h} \leqslant C_{17} b^{h} y_{h+1}^{1-\varepsilon}, \quad h=0,1,2, \ldots,
$$

с какими-либо положительными постоянными $C_{17}, \varepsilon>0$ u $b<1$. Тогда, если

$$
y_{h} \leqslant C_{18} \text { сразу для всех } h, \text { то } y_{0}^{\varepsilon} \leqslant C_{19} .
$$

Для любой локально суммируемой функции $f(z)$ обозначим

$$
\|\mid f\|_{r}=\sup _{\rho \geqslant r}\left(\rho^{-\frac{K}{\lambda-1}} \int_{B_{\rho}}|f| d x d y\right) .
$$

Поскольку решение задачи (1.1), (1.2) является субрешением задачи без демпфирования при $a(\rho)=f(t)=0$, то (см. [8]) справедливо

ПредЛОЖЕНИЕ 7. Пусть $u_{0} \in L_{1, \operatorname{loc}}\left(R^{N+M}\right)$ и для некоторого заданного $r>0$

$$
\left\|\left|u_{0} \|\right|_{r}<\infty\right.
$$

Тогда существуют такие положительные постоянные $C_{20}, C_{21}, C_{22}, C_{23}$, зависящие лищь от параметров $\lambda, N, M, \alpha$, что для решения (обобщенного) задачи $(1.1),(1.2)$ при всех $0<t<T_{r}$ u $R>r$, где $T_{r}=\left.C_{20}\left\|u_{0}\right\|\right|_{r} ^{-\frac{1}{\lambda-1}}$, cnраведливы оченки

$$
\begin{gathered}
\left.\left\|\left|u(t)\left\|\left.\right|_{r} \leqslant C_{21}\right\|\right| u_{0}\right\|\right|_{r}, \\
\|u(t)\|_{L_{\infty}\left(B_{R}\right)} \leqslant C_{22} t^{-\frac{Q}{K}} R^{\frac{\lambda+1}{\lambda-1}}\left\|\left|u_{0} \|\right|_{r}^{\frac{\lambda+1}{K}} .\right.
\end{gathered}
$$

Пусть $u_{0} \in L_{1}\left(R^{N+M}\right) u \operatorname{supp} u_{0} \subset B_{R_{0}}, R_{0}<\infty$. Тогда для всех $t>0$

$$
Z(t)=\inf \left\{\rho: u(t)=0 \text { для n.в. }(x, y) \in R^{N+M} \backslash B_{\rho}\right\} \leqslant 4 R_{0}+C_{23} t^{\frac{1}{K}}\left\|u_{0}\right\|_{1}^{\frac{\lambda-1}{K}} .
$$

ПРЕДЛОЖЕНИЕ 8 (см. [8; лемма 3.3]). Если предположения предложения 7 выполняются, то для любого $t \in(0, T)$ u $R \geqslant r$

$$
\|u(t)\|_{\infty, B_{R} \times(t / 2, t)} \leqslant \frac{C_{24}}{t^{\frac{Q}{K}}}\left(f_{t / 4}^{t} \int_{B_{2 R}} u d x\right)^{\frac{\lambda+1}{K}} .
$$

\section{§ 3. Доказательство теоремы 1}

Прежде всего докажем существование решения задачи (1.1), (1.2). Рассмотрим последовательность задач

$$
\begin{gathered}
\frac{\partial u_{n}}{\partial t}=\operatorname{div}_{L}\left(\left|D_{L} u_{n}\right|^{\lambda-1} D_{L} u_{n}\right)-\min \left\{a(\rho(z)) f(t)\left|D_{L} u_{n}^{\nu}\right|^{q}, n\right\}, \\
(z, t) \in S_{T, n}=B_{n} \times(0, T), \quad B_{n} \equiv\left\{z \in \mathbb{R}^{N+M}: \rho(z, 0) \leqslant n\right\} \\
u_{n}(z, t)=0 \quad \text { на } \partial B_{n} \times(0, T), \\
u_{n}(z, 0)=u_{0 n}(z) \in C_{0}^{\infty}\left(B_{n}\right), \quad u_{0 n}(z) \geqslant 0, \quad \text { где }\left\|u_{0 n}\right\|_{1, \mathbb{R}^{N+M}} \leqslant\left\|u_{0}\right\|_{1, \mathbb{R}^{N+M}}, \\
\lim _{n \rightarrow \infty} \int_{\mathbb{R}^{N+M}} u_{0 n} \eta(z) d z=\int_{\mathbb{R}^{N+M}} u_{0} \eta(z) d z \quad \forall \eta(z) \in C_{0}^{\infty}\left(\mathbb{R}^{N+M}\right) .
\end{gathered}
$$


Методом монотонности и компактности Лионса [33] для каждого фиксированного $n$ доказывается, что существует решение $u_{n}$ из класса $C\left(0, T ; L_{2}\left(B_{n}\right)\right) \cap$ $L_{\lambda+1}\left(0, T ; \mathfrak{L}_{1, \lambda+1}\left(B_{n}\right)\right)$. Теперь докажем, что решения $u_{n}$ задачи $(3.1)-(3.3)$ сходятся к решению исходной задачи Коши. Необходимо перейти к пределу в интегральном тождестве для $u_{n}$ при $n \rightarrow \infty$. Отметим, что наличие демпфирующего слагаемого в уравнении делает такой предельный переход нетривиальным. Ниже мы будем следовать схеме, предложенной в работах [34], [35]. Для этой цели нам потребуется сходимость почти всюду градиентов решения. Мы докажем сильную сходимость градиентов в $L_{\lambda+1}\left(0, T ; \mathfrak{L}_{1, \lambda+1, \operatorname{loc}}\left(\mathbb{R}^{N+M}\right)\right)$. Выберем произвольное компактное множество $\mathfrak{K} \subset S_{T}$. Существует такое $n_{0}$, что $\mathfrak{K} \subset(0, T) \times B_{n_{0}}$. Прежде всего покажем, что для всех $u_{n}$ выполняется неравенство

$$
\int_{B_{n_{0}}} u_{n}(t) d z \leqslant \int_{B_{n_{0}}} u_{0 n} d z
$$

Умножим уравнение (3.1) на финитную функцию $u_{n} /\left(u_{n}+\varepsilon\right)$, где $\varepsilon>0$. Интегрируя по частям, получим

$$
\begin{aligned}
\int_{B_{n_{0}}} \int_{0}^{u_{n}(t)} & \frac{s}{s+\varepsilon} d s d z+\varepsilon \int_{0}^{t} \int_{B_{n_{0}}} \frac{\left|D_{L} u_{n}\right|^{\lambda+1}}{\left(u_{n}+\varepsilon\right)^{2}} d z d \tau \\
& +\int_{0}^{t} \int_{B_{n_{0}}} \frac{a(z) f(\tau)\left|D_{L} u_{n}^{\nu}\right|^{q} u_{n}}{u_{n}+\varepsilon} d z d \tau \leqslant \int_{B_{n_{0}}} \int_{0}^{u_{n 0}} \frac{s}{s+\varepsilon} d s d z .
\end{aligned}
$$

Переходя к пределу при $\varepsilon \rightarrow 0$ получим полезное и в дальнейшем неравенство

$$
\int_{B_{n_{0}}} u_{n}(t) d z+\int_{0}^{t} \int_{B_{n_{0}}} a(z) f(\tau)\left|D_{L} u_{n}^{\nu}\right|^{q} d z d \tau \leqslant \int_{B_{n_{0}}} u_{0 n} d z .
$$

Равномерная ограниченность для $u_{n}$ следует из предложения 8 и в силу (3.4):

$$
\left\|u_{n}(\cdot, t)\right\|_{\infty, B_{n}} \leqslant C t^{-\frac{Q}{K}}\left\|u_{0}\right\|_{1, \mathbb{R}^{N+M}}^{\frac{\lambda+1}{K}} \quad \forall t>0 .
$$

Очевидно, что добавление к уравнению положительного демпфирующего слагаемого не приводит к выходу решения из гёльдеровского класса. Поскольку константа Гёльдера зависит от $\left\|u_{n}\right\|_{\infty}$, которая в силу (3.5) не зависит от $n$, то на компакте $\mathfrak{K}$ последовательность $\left\{u_{n}\right\}$ локально непрерывна по Гёльдеру сразу для всех $n$. Следовательно, можно извлечь равномерно сходящуюся к $u$ подпоследовательность, которую мы снова обозначим $\left\{u_{n}\right\}, n \geqslant n_{0}$. Кроме того, для $\left\{u_{n}\right\}, n \geqslant n_{0}$, справедлива еще одна априорная оценка

$$
\begin{aligned}
& \sup _{0<t<T} \int_{B_{n_{0}}} u_{n}^{2}(t) d z+\iint_{S_{T, n_{0}}}\left|D_{L} u_{n}\right|^{\lambda+1} d z d \tau \\
&+\iint_{S_{T_{, n}}} \min \left\{a(\rho(z)) f(t)\left|D_{L} u_{n}^{\nu}\right|^{q}, n\right\} u_{n} d z d \tau \leqslant \int_{B_{n_{0}}} u_{0 n}^{2} d z .
\end{aligned}
$$

Откуда следует

$$
\iint_{\mathfrak{K}}\left|D_{L} u_{n}\right|^{\lambda+1} d z d \tau \leqslant C\left(\mathfrak{K}, u_{0}\right) .
$$

Используя равномерную сходимость $\left\{u_{n}\right\}$, докажем фундаментальность последовательности $\left\{D_{L} u_{n}\right\} \in L_{\lambda+1}\left(0, T ; \mathfrak{L}_{1, \lambda+1}(\mathfrak{K})\right)$. Выберем два произвольных 
члена последовательности $\left\{u_{n}\right\}: u_{j}$ и $u_{k}, k, j>n_{0}$. Тогда для разности $u_{j}-u_{k}$ имеем

$$
\begin{aligned}
& \frac{\partial\left(u_{j}-u_{k}\right)}{\partial t}-\left(\operatorname{div}_{L}\left(\left|D_{L} u_{j}\right|^{\lambda-1} D_{L} u_{j}\right)-\operatorname{div}_{L}\left(\left|D_{L} u_{k}\right|^{\lambda-1} D_{L} u_{k}\right)\right) \\
& \quad+\min \left\{a(\rho(z)) f(t)\left|D_{L} u_{j}^{\nu}\right|^{q}, j\right\}-\min \left\{a(\rho(z)) f(t)\left|D_{L} u_{k}^{\nu}\right|^{q}, k\right\}=0 .
\end{aligned}
$$

Умножим полученное уравнение на $\left(u_{j}-u_{k}\right) \xi^{2}$, где $\xi(z, \tau)-$ стандартная срезающая функция, финитная на всем цилиндре $S_{T, n_{0}}$, такая, что $0 \leqslant \xi \leqslant 1$,

$$
\begin{gathered}
\xi=1 \quad \text { на } B_{R / 2}, \quad \xi=0 \text { на } B_{R}, \\
\xi(z, \tau)=0 \quad \text { при } \tau \leqslant \frac{t}{2}, \quad \xi(z, \tau)=1 \quad \text { при } \tau \geqslant t, \\
\left|D_{L} \xi\right| \leqslant \frac{C}{R}, \quad 0 \leqslant D_{\tau} \xi \leqslant \frac{C}{\tau} .
\end{gathered}
$$

Пользуясь сильной монотонностью оператора $A(u)=-\operatorname{div}_{L}\left(\left|D_{L} u\right|^{\lambda-1} D_{L} u\right)$ и неравенством Гёльдера, получаем

$$
\begin{aligned}
& \iint_{S_{T, n_{0}}}\left|D_{L} u_{j}-D_{L} u_{k}\right|^{\lambda+1} \xi^{2} d z d \tau \leqslant C \iint_{S_{T, n_{0}}}\left|u_{j}-u_{k}\right|^{2} \xi\left|\xi_{\tau}\right| d z d \tau \\
& \quad+C \iint_{S_{T, n_{0}}}\left(\left|D_{L} u_{j}\right|^{\lambda}+\left|D_{L} u_{k}\right|^{\lambda}\right) \xi\left|D_{L} \xi\right|\left|u_{j}-u_{k}\right| d z d \tau \\
& \quad+\left.C \iint_{S_{T, n_{0}}}|a(\rho(z)) f(t)| D_{L} u_{j}^{\nu}\right|^{q}+a(\rho(z)) f(t)\left|D_{L} u_{k}^{\nu}\right|^{q}|| u_{j}-u_{k} \mid \xi^{2} d z d \tau \\
& \quad \leqslant \iint_{S_{T, n_{0}}}\left|u_{j}-u_{k}\right|^{2} \xi\left|\xi_{\tau}\right| d z d \tau \\
& \quad+C\left(\iint_{S_{T, n_{0}}}\left|D_{L} u_{j}\right|^{\lambda+1} d z d \tau\right)^{\frac{\lambda}{\lambda+1}}\left(\iint_{S_{T, n_{0}}}\left|u_{j}-u_{k}\right|^{\lambda+1} \xi^{\lambda+1}\left|D_{L} \xi\right|^{\lambda+1} d z d \tau\right)^{\frac{1}{\lambda+1}} \\
& \quad+C\left(\iint_{S_{T, n_{0}}}\left|D_{L} u_{k}\right|^{\lambda+1} d z d \tau\right)^{\frac{\lambda}{\lambda+1}}\left(\iint_{S_{T, n_{0}}}\left|u_{j}-u_{k}\right|^{\lambda+1} \xi^{\lambda+1}\left|D_{L} \xi\right|^{\lambda+1} d z d \tau\right)^{\frac{1}{\lambda+1}} \\
& \quad+\left(\int_{B_{n_{0}}} u_{0 j} d z+\int_{B_{n_{0}}} u_{0 k} d z\right)_{B_{n_{0}} \times(t / 2, T)} \sup \left|u_{j}-u_{k}\right|^{2} \rightarrow 0 \quad \text { при } j, k \rightarrow \infty .
\end{aligned}
$$

Переходя к пределу, мы использовали равномерную сходимость $\left\{u_{n}\right\}$, а также оценки (3.4) и (3.7). Теперь можно выделить подпоследовательность, для которой

$$
D_{L} u_{n} \rightarrow D_{L} u \quad \text { п.в. на любом компакте } \mathfrak{K},
$$

$$
D_{L} u_{n} \rightarrow D_{L} u \quad \text { сильно в } L_{\lambda+1}\left(0, T ; \mathfrak{L}_{1, \lambda+1}\left(B_{n_{0}}\right)\right) \text {. }
$$

В интегральном тождестве для $u_{n}$ можно перейти к пределу. Таким образом, можно считать доказанным, что $u_{n}$ стремится равномерно к решению уравнения (1.1) на любом компакте внутри $S_{T}$. Перейдя к пределу при $n \rightarrow \infty$, видим, что оценки (3.4), (3.5) справедливы и для $u$. Чтобы доказать, что слабое решение уравнения является слабым решением задачи Коши (1.1), (1.2), 
нам необходимо проследить поведение $u$ при $t \rightarrow 0$. Очевидно, что оценка (3.5) не дает равномерной ограниченности при $t \rightarrow 0$. Поэтому мы не можем использовать равномерную сходимость $\left\{u_{n}\right\}$, как раньше. Сначала получим вспомогательную оценку. Умножим уравнение $(1.1)$ на $t^{\beta} u^{\theta} \xi^{\lambda+1}(z) ; R, \beta, \theta>0$ выберем позднее. В качестве срезающей возьмем функцию из пространства $C_{0}^{1}\left(\mathbb{R}^{N+M}\right)$ такую, что

$$
\xi(z)=\left\{\begin{array}{ll}
0 & \text { при } \rho(z)>R, \\
1 & \text { при } \rho(z)<\frac{R}{2},
\end{array} \quad\left|D_{L} \xi\right| \leqslant C \frac{2}{R} .\right.
$$

Проинтегрировав по $B_{R} \times(0, t)$ стандартным способом, получим

$$
\begin{aligned}
& \frac{1}{\theta+1} \int_{B_{R}} t^{\beta} \xi^{\lambda+1}(z) u^{\theta+1}(z, t) d z+\theta \iint_{S_{t, R}} \tau^{\beta} \xi^{\lambda+1} u^{\theta-1}\left|D_{L} u\right|^{\lambda+1} d z d \tau \\
& +(\lambda+1) \iint_{S_{t, R}} \tau^{\beta} \xi^{\lambda} u^{\theta}\left|D_{L} u\right|^{\lambda-1} D_{L} u D_{L} \xi d z d \tau \\
& \quad+\iint_{S_{t, R}} a(\rho(z)) f(\tau)\left|D_{L} u^{\nu}\right|^{q} \tau^{\beta} \xi^{\lambda+1} u^{\theta} d z d \tau \\
& =\frac{\beta}{\theta+1} \iint_{S_{t, R}} \tau^{\beta-1} \xi^{\lambda+1}(z) u^{\theta+1}(z, t) d z d \tau
\end{aligned}
$$

Используя неравенство Юнга и неравенство (3.5), оценим

$$
\begin{aligned}
& \frac{1}{\theta+1} \int_{B_{R}} t^{\beta} \xi^{\lambda+1}(z) u^{\theta+1}(z, t) d z+\theta \iint_{S_{t, R}} \tau^{\beta} \xi^{\lambda+1} u^{\theta-1}\left|D_{L} u\right|^{\lambda+1} d z d \tau \\
& \quad+\iint_{S_{t, R}} a(\rho(z)) f(\tau)\left|D_{L} u^{\nu}\right|^{q} t^{\beta} \xi^{\lambda+1} u^{\theta} d z d \tau \\
& \quad \leqslant C \iint_{S_{t, R}} \tau^{\beta-1} u^{\theta+1}\left(1+\tau R^{-(\lambda+1)} u^{\lambda-1}\right) d z d \tau \leqslant C \iint_{S_{t, R}} \tau^{\beta-1} u^{\theta+1} d z d \tau .
\end{aligned}
$$

Таким образом, если $\theta=(\lambda-1) / \lambda, 0<\beta<1 / \lambda, R$ фиксировано, получена оценка

$$
\iint_{S_{t, R}} \tau^{\beta} u^{-\frac{1}{\lambda}}\left|D_{L} u\right|^{\lambda+1} d z d \tau \leqslant C \iint_{S_{t, 2 R}} \tau^{\beta-1} u^{\frac{\lambda-1}{\lambda}+1} d z d \tau .
$$

Последний интеграл можно оценить, пользуясь предложением 8 и неравенством (3.5):

$$
\begin{aligned}
& \iint_{S_{t, R}} \tau^{\beta-1} u^{\frac{\lambda-1}{\lambda}+1} d z d \tau \leqslant\left(\sup _{0<\tau<t} \int_{B_{R}} u d z\right) \int_{0}^{t} \tau^{\beta-1}\|u\|_{\infty, B_{R}}^{\frac{\lambda-1}{\lambda}} d \tau \\
& \quad \leqslant C t^{\beta-\frac{Q(\lambda-1)}{K \lambda}}\left(\sup _{0<\tau<t} \int_{B_{R}} u d z\right)^{1+\frac{(\lambda+1)(\lambda-1)}{K \lambda}} \leqslant C t^{\beta-\frac{Q(\lambda-1)}{K \lambda}}\left\|u_{0}\right\|_{1}^{1+\frac{(\lambda+1)(\lambda-1)}{K \lambda}} .
\end{aligned}
$$

Теперь можно непосредственно доказать, что $u$ есть решение исходной задачи. Для произвольного компакта $\mathfrak{K} \subset \mathbb{R}^{N+M}$ найдется $R>0$ такое, что $\mathfrak{K} \subset B_{R / 2}$. 
Умножив уравнение (1.1) на срезку $\xi$, определенную в (3.10), и проинтегрировав по $B_{R} \times(0, t)$ стандартным способом, получим

$$
\begin{gathered}
\int_{B_{R}} u(t) \xi d z+\iint_{S_{t, R}}\left|D_{L} u\right|^{\lambda-1} D_{L} u D_{L} \xi d z d \tau \\
+\iint_{S_{t, R}} a(\rho(z)) f(\tau)\left|D_{L} u^{\nu}\right|^{q} \xi d z d \tau=\int_{B_{R}} u_{0} \xi d z \\
\left|\int_{B_{R}} u(t) \xi d z-\int_{B_{R}} u_{0} \xi d z\right| \\
\leqslant \frac{C}{R} \iint S_{t, R}\left|D_{L} u\right|^{\lambda} d z d \tau+\iint_{S_{t, R}} a(\rho(z)) f(\tau)\left|D_{L} u^{\nu}\right|^{q} \xi d z d \tau \equiv \frac{C}{R} I_{1}+I_{2} .
\end{gathered}
$$

Оценим последние интегралы

$$
\begin{aligned}
I_{1} & =\iint_{S_{t, R}}\left|D_{L} u\right|^{\lambda} d z d \tau \\
& \leqslant\left(\iint_{S_{t, R}} \tau^{\beta} u^{-\frac{1}{\lambda}}\left|D_{L} u\right|^{\lambda+1} d z d \tau\right)^{\frac{\lambda}{\lambda+1}}\left(\iint_{S_{t, R}} \tau^{-\beta \lambda} u d z d \tau\right)^{\frac{1}{\lambda+1}} \\
& \leqslant\left(C t^{\beta-\frac{Q(\lambda-1)}{K \lambda}}\left(\sup _{0<\tau<t} \int_{B_{2 R}} u d z\right)^{1+\frac{(\lambda+1)(\lambda-1)}{K \lambda}}\right)^{\frac{\lambda}{\lambda+1}}\left(t^{1-\beta \lambda}\left(\sup _{0<\tau<t} \int_{B_{2 R}} u d z\right)\right)^{\frac{1}{\lambda+1}} \\
& \leqslant C t^{\frac{1}{K}}\left(\sup _{0<\tau<t} \int_{B_{2 R}} u d z\right)^{1+\frac{\lambda-1}{K}} \leqslant \frac{C}{R} t^{\frac{1}{K}}\left\|u_{0}\right\|_{1}^{1+\frac{\lambda-1}{K}} \rightarrow 0 \quad \text { при } t \rightarrow 0 .
\end{aligned}
$$

Интеграл $I_{2}$ ограничен сверху (см. (3.5)) и стремится к нулю вместе с $t$. Следовательно, (1.2) доказано.

Переходим к доказательству оценки (1.10).

Лемма 3.1. Пусть и-неотрицательное решение уравнения (1.1), $\operatorname{supp} u_{0} \subset$ $B_{R_{0}}, R_{0}<\infty$, тогда для любых $0 \leqslant t_{1} \leqslant t_{2}$ u $R>2 R_{0}, R_{i}=R\left(1-2^{-i-1}\right)$, $\tilde{R}_{i}=\left(R_{i}+R_{i+1}\right) / 2, R_{i}<\tilde{R}_{i}, U_{i}=\left\{\rho(z)>R_{i}\right\}, \tilde{U}_{i}=\left\{\rho(z)>\tilde{R}_{i}\right\}$ справедливо неравенство

$$
\begin{aligned}
\sup _{t_{1}<\tau<t_{2}} \int_{\tilde{U}_{i}} u^{\theta+1} d z+\int_{t_{1}}^{t_{2}} \int_{\tilde{U}_{i}} u^{\theta-1}\left|D_{L} u\right|^{\lambda+1} d z d \tau \\
\quad+\int_{t_{1}}^{t_{2}} \int_{\tilde{U}_{i}} a(\rho(z)) f(\tau) u^{\theta}\left|D_{L} u^{\nu}\right|^{q} d z d \tau \leqslant C \frac{2^{i(\lambda+1)}}{R^{\lambda+1}} \int_{t_{1}}^{t_{2}} \int_{U_{i} \backslash \tilde{U}_{i}} u^{\lambda+\theta} d z d \tau .
\end{aligned}
$$

Доказательство. Умножим уравнение на $u^{\theta} \xi_{i}^{\lambda+1}(z) ; \theta>0$ выберем позднее. Выберем в качестве срезающей функцию из пространства $C^{1}\left(\mathbb{R}^{N+M}\right)$ такую, что

$$
\xi_{i}(z)=\left\{\begin{array}{ll}
0 & \text { при } \rho(z)<R_{i}, \\
1 & \text { при } \rho(z)>\tilde{R}_{i},
\end{array} \quad\left|D_{L} \xi_{i}\right| \leqslant C \frac{2^{i}}{R} .\right.
$$

Проинтегрировав по $\tilde{U}_{i} \times\left(0, t_{2}\right)$ стандартным способом, используя неравенство Юнга, получим требуемое неравенство. Заметим, что отсутствие в неравенстве 
члена $\sup \int_{\tilde{U}_{i}} u_{0}^{\theta+1} d z$ объясняется тем, что интегрирование идет по области, в которой не содержится носитель $u_{0}$.

ЛЕмма 3.2. Пусть и - неотрицательное решение уравнения (1.1),

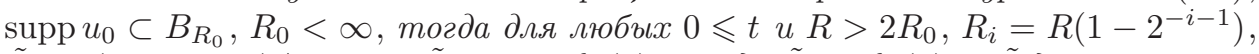
$\tilde{R}_{i}=\left(R_{i}+R_{i+1}\right) / 2, R_{i}<\tilde{R}_{i}, U_{i}=\left\{\rho(z)>R_{i}\right\}, \tilde{U}_{i}=\left\{\rho(z)>\tilde{R}_{i}\right\}$ справедливо неравенство

$$
y_{i+1} \leqslant C \frac{2^{i(\lambda+1)}}{R^{\lambda+1}} t^{\frac{(1+\theta)(\lambda+1)}{K_{1+\theta}}} y_{i}^{1+\frac{(\lambda-1)(\lambda+1)}{K_{1+\theta}}},
$$

где

$$
\begin{aligned}
y_{i+1} \equiv \sup _{0<\tau<t} \int_{\tilde{U}_{i+1}} u^{\theta+1} d z+\int_{0}^{t} \int_{\tilde{U}_{i+1}} u^{\theta-1}\left|D_{L} u\right|^{\lambda+1} d z d \tau \\
\quad+\int_{0}^{t} \int_{\tilde{U}_{i+1}} a(\rho(z)) f(\tau) u^{\theta}\left|D_{L} u^{\nu}\right|^{q} d z d \tau+\frac{2^{i(\lambda+1)}}{R^{\lambda+1}} \int_{0}^{t} \int_{U_{i+1} \backslash \tilde{U}_{i+1}} u^{\lambda+\theta} d z d \tau .
\end{aligned}
$$

ДокАЗАтЕЛЬство. Пусть

$$
\bar{\xi}_{i+1}(z)=\left\{\begin{array}{ll}
0 & \text { при } \rho(z)<\tilde{R}_{i}, \\
1 & \text { при } \rho(z)>R_{i+1},
\end{array} \quad\left|D_{L} \bar{\xi}_{i}\right| \leqslant C \frac{2^{i}}{R} .\right.
$$

В неравенстве (2.1) положим $f=v_{i+1}=\left(u \bar{\xi}_{i+1}(z)\right)^{\omega}, \omega=(\lambda+\theta) /(\lambda+1)$, $\beta_{1}=Q(\lambda-1) / K_{1+\theta}, \beta_{2}=(\theta+1) / \omega>1$. Проинтегрируем неравенство (2.1) от 0 до $t$ по $d \tau$ :

$$
\begin{aligned}
& \int_{0}^{t} \int_{U_{i+1} \backslash \tilde{U}_{i+1}} u^{\lambda+\theta} d z d \tau=\int_{0}^{t} \int_{U_{i+1} \backslash \tilde{U}_{i+1}} v_{i+1}^{\lambda+1} d z d \tau \\
& \quad \leqslant C \int_{0}^{t}\left(\int_{\mathbb{R}^{N+M}}\left|D_{L} v_{i+1}\right|^{\lambda+1} d z\right)^{\beta_{1}}\left(\int_{\tilde{U}_{i}} v_{i+1}^{\beta_{2}} d z\right)^{\frac{\left(1-\beta_{1}\right)(\lambda+1)}{\beta_{2}}} d \tau \\
& \quad \leqslant C t^{1-\beta_{1}}\left(\int_{0}^{t} \int_{\mathbb{R}^{N+M}}\left|D_{L} v_{i+1}\right|^{\lambda+1} d z d \tau\right)^{\beta_{1}}\left(\sup _{0<\tau<t} \int_{\tilde{U}_{i}} v_{i+1}^{\beta_{2}} d z\right)^{\frac{\left(1-\beta_{1}\right)(\lambda+1)}{\beta_{2}}} \\
& \quad \leqslant C t^{1-\beta_{1}} y_{i}^{\beta_{1}+\frac{\lambda+1}{\beta_{2}}\left(1-\beta_{1}\right)}=C t^{1-\beta_{1}} y_{i}^{1+\left(1-\beta_{1}\right)\left(\frac{\lambda+1}{\beta_{2}}-1\right)} .
\end{aligned}
$$

Применив лемму 3.1, получим требуемую оценку.

Используем демпфирующую часть уравнения, чтобы получить вспомогательное рекурсивное неравенство.

ЛЕмма 3.3. Пусть выполнены условия леммы 3.2 и

$$
Z(t)=\inf \left\{\rho: u(\cdot, t)=0 \text { для п.в. } z \in \mathbb{R}^{N+M} \backslash B_{\rho}\right\} .
$$

Тогда справедливо неравенство

$$
y_{i+1} \leqslant C \frac{2^{i(\lambda+1)}}{R^{\lambda+1}} R^{Q \frac{\nu q-\lambda}{\nu q+\theta}-q \frac{\lambda+\theta}{\nu q+\theta}} \frac{t^{\frac{\nu q-\lambda}{\nu q+\theta}}}{f(t)^{\frac{\lambda+\theta}{\nu q+\theta}}}\left(\frac{Z(t)^{2 q}}{a(Z(t))}\right)^{\frac{\lambda+\theta}{\nu q+\theta}} y_{i}^{\frac{\lambda+\theta}{\nu q+\theta}} .
$$


ДокАЗАТЕльство. Пусть $\omega=u^{\frac{\nu q+\theta}{q}}$. В силу предложения 7 ясно, что $Z(t)$ конечно для каждого $t$. Следовательно, $\omega \in \stackrel{\circ}{\mathfrak{L}}_{1, p}\left(B_{Z(t)}\right)$ для каждого $t>0$. Используя обозначения и результаты лемм 3.1 и $3.2,(2.2)$, неравенство Гёльдера и свойства весовых функций $a(\rho)$ и $f(t)$, получаем

$$
\begin{aligned}
y_{i+1} \leqslant & C \frac{2^{i(\lambda+1)}}{R^{\lambda+1}} \int_{0}^{t} \int_{U_{i+1} \backslash \tilde{U}_{i+1}} u^{\lambda+\theta} d z d \tau \\
\leqslant & C \frac{2^{i(\lambda+1)}}{R^{\lambda+1}} \int_{0}^{t}\left(\int_{B_{Z(t)} \backslash B_{R_{i+1}}} \omega^{q} d z\right)^{\frac{\lambda+\theta}{\nu q+\theta}} \operatorname{mes}\left\{U_{i+1} \backslash \tilde{U}_{i+1}\right\}^{\frac{\nu q-\lambda}{\nu q+\theta}} d \tau \\
\leqslant & C \frac{2^{i(\lambda+1)}}{R^{\lambda+1}} R_{i+1}^{-q \frac{\lambda+\theta}{\nu q+\theta}} \\
& \times \int_{0}^{t}\left(\frac{Z(t)^{2 q}}{a(Z(t)) f(\tau)} \int_{B_{Z(t)} \backslash B_{R_{i+1}}} a(\rho(z)) f(\tau)\left|D_{L} \omega\right|^{q} d z\right)^{\frac{\lambda+\theta}{\nu q+\theta}} \tilde{R}_{i}^{Q \frac{\nu q-\lambda}{\nu q+\theta}} d \tau \\
\leqslant & C \frac{2^{i(\lambda+1)}}{R^{\lambda+1}} R^{Q \frac{\nu q-\lambda}{\nu q+\theta}-q \frac{\lambda+\theta}{\nu q+\theta}}\left(\frac{Z(t)^{2 q}}{a(Z(t))}\right)^{\frac{\lambda+\theta}{\nu q+\theta}} y_{i}^{\frac{\lambda+\theta}{\nu q+\theta}}\left(\int_{0}^{t}\left[\frac{1}{f(\tau)}\right]^{\frac{\lambda+\theta}{\nu q-\lambda}} d \tau\right)^{\frac{\nu q-\lambda}{\nu q+\theta}} \\
\leqslant & C \frac{2^{i(\lambda+1)}}{R^{\lambda+1}} R^{Q \frac{\nu q-\lambda}{\nu q+\theta}-q \frac{\lambda+\theta}{\nu q+\theta}} \frac{t^{\frac{\nu q-\lambda}{\nu q+\theta}}}{f(t)^{\frac{\lambda+\theta}{\nu q+\theta}}}\left(\frac{Z(t)^{2 q}}{a(Z(t))}\right)^{\frac{\lambda+\theta}{\nu q+\theta}} y_{i}^{\frac{\lambda+\theta}{\nu q+\theta}} \cdot
\end{aligned}
$$

Здесь появляется условие на $\theta$. Выберем его достаточно малым, чтобы выполнялось неравенство $-\mu \frac{\lambda+\theta}{\nu q-\lambda}+1>0$.

Лемма 3.4. Если существует последовательность $\left\{y_{i}\right\}$ такая, что

$$
y_{i+1} \leqslant C 2^{i(\lambda+1)} A y_{i}^{\frac{1}{a}}, \quad a<1, \quad y_{i+1} \leqslant C 2^{i(\lambda+1)} B y_{i}^{\frac{1}{b}}, \quad b>1,
$$

и если достаточно мала величина

$$
\left(y_{0} B^{b}\right)^{\frac{1-a}{b}} A^{a} \leqslant C^{-\frac{1}{(1-a) / b}} 2^{-\frac{(\lambda+1)(a+b)}{((1-a) / b)^{2}}} \equiv C_{0},
$$

где $C_{0}<1$ - фиксированное число, тогда $y_{i} \rightarrow 0$ при $i \rightarrow \infty$.

ДокАЗАТЕЛЬСтво. По условию леммы имеем

$$
y_{i+1}^{a} \leqslant C 2^{i(\lambda+1) a} A^{a} y_{i}, \quad y_{i+1}^{b} \leqslant C 2^{i(\lambda+1) b} B^{b} y_{i},
$$

откуда следует, что

$$
\frac{y_{i+1}^{a}}{A^{a}}+\frac{y_{i+1}^{b}}{B^{b}} \leqslant C 2^{i(\lambda+1)(a+b)} y_{i} .
$$

Используя неравенство Юнга с показателями

$$
\frac{1}{p}=\frac{b}{b+1-a}, \quad \frac{1}{p^{\prime}}=\frac{1-a}{b+1-a},
$$

получим

$$
\frac{y_{i+1}^{\frac{b}{b+1-a}}}{A^{\frac{a b}{b+1-a}} B^{\frac{b(1-a)}{b+1-a}}}=\frac{y_{i+1}^{a \frac{1}{p}} y_{i+1}^{b \frac{1}{p^{\prime}}}}{A^{a \frac{1}{p}} B^{b \frac{1}{p^{\prime}}}} \leqslant \frac{y_{i+1}^{a}}{A^{a}}+\frac{y_{i+1}^{b}}{B^{b}} .
$$

Если обозначить $b /(b+1-a)=\varepsilon_{1}<1$, тогда

$$
\frac{y_{i+1}^{\varepsilon_{1}}}{A^{a \varepsilon_{1}} B^{b\left(1-\varepsilon_{1}\right)}} \leqslant C 2^{i(\lambda+1)(a+b)} y_{i}
$$


и по итерационной лемме (предложение 5), если выполняется (3.20), имеет место стремление к нулю.

Введем вспомогательные обозначения

$$
\begin{gathered}
A \equiv R^{-(\lambda+1)} t^{\frac{(1+\theta)(\lambda+1)}{K_{1+\theta}}}, \quad a=\frac{1}{1+(\lambda-1)(\lambda+1) / K_{1+\theta}}<1, \\
B \equiv R^{Q \frac{\nu q-\lambda}{\nu q+\theta}-(\lambda+1)-q \frac{\lambda+\theta}{\nu q+\theta}} \frac{t^{\frac{\nu q-\lambda}{\nu q+\theta}}}{f(t)^{\frac{\lambda+\theta}{\nu q+\theta}}}\left(\frac{Z(t)^{2 q}}{a(Z(t))}\right)^{\frac{\lambda+\theta}{\nu q+\theta}}, \quad b=\frac{\nu q+\theta}{\lambda+\theta}>1 .
\end{gathered}
$$

Лемма 3.5. Пусть выполненъ условия лемм 3.1, 3.2, тогда условие (3.20) справедливо, если

$$
\left(\frac{Z(t)^{2 q}}{a(Z(t))}\right)^{\lambda-1} R^{-2 q(\lambda-1)-H} \frac{t^{\nu q-\lambda}}{f(t)^{\lambda-1}} \leqslant \delta,
$$

где $\delta$ - фиксированное число и достаточно мало.

ДокАЗАТЕЛЬство. Из лемм 3.3, 3.2 следует, что (3.19) выполнено. В формулировке 3.1 положим для произвольного $\hat{R}: \hat{R} \geqslant 2 R \geqslant 4 R_{0}$

$$
\tilde{R}_{i}=\hat{R}_{i}, \quad R_{i}=\hat{R}_{i+1}, \quad R_{i}<\tilde{R}_{i}, \quad \text { где } \quad \hat{R}_{i}=\frac{\hat{R}}{4}\left(1+2^{-i}\right), \quad t_{1}=0, \quad t_{2}=t .
$$

Лемма 3.1 дает

$$
\begin{gathered}
\sup _{0<\tau<t} \int_{\rho \geqslant \hat{R}_{i}} u^{\theta+1} d z+\int_{0}^{t} \int_{\rho \geqslant \hat{R}_{i}} u^{\theta-1}\left|D_{L} u\right|^{\lambda+1} d z d \tau \\
+\int_{0}^{t} \int_{\rho \geqslant \hat{R}_{i}} a(\rho(z)) f(\tau) u^{\theta}\left|D_{L} u^{\nu}\right|^{q} d z d \tau \\
\leqslant C \frac{2^{i(\lambda+1)}}{R^{\lambda+1}} \int_{0}^{t} \int_{\hat{R}_{i+1} \leqslant \rho \leqslant \hat{R}_{i}} u^{\lambda+\theta} d z d \tau
\end{gathered}
$$

Повторим доказательство леммы 3.3 до некоторой степени. Пусть $\omega=u^{\frac{\nu q+\theta}{q}} \in$ $\stackrel{\circ}{\mathfrak{L}}_{1, p}\left(B_{Z(t)}\right)$ для каждого $t>0$. Оценим правую часть $(3.23)$, используя неравенство Гёльдера и неравенство Пуанкаре (2.2)

$$
\begin{aligned}
& \int_{0}^{t} \int_{\hat{R}_{i+1} \leqslant \rho \leqslant \hat{R}_{i}} u^{\lambda+\theta} d z d \tau=\int_{0}^{t} \int_{\hat{R}_{i+1} \leqslant \rho \leqslant \hat{R}_{i}} \omega^{\frac{(\lambda+\theta) q}{\nu q+\theta}} d z d \tau \\
& \leqslant C \int_{0}^{t}\left(\frac{Z(t)^{2 q}}{a(Z(t)) f(\tau)} \int_{\hat{R}_{i+1} \leqslant \rho \leqslant Z(t)} a(\rho(z)) f(\tau)\left|D_{L} \omega\right|^{q} d z\right)^{\frac{\lambda+\theta}{\nu+\theta}} \hat{R}_{i+1}^{-q \frac{\lambda+\theta}{\nu q+\theta}} \hat{R}_{i}^{Q \frac{\nu q-\lambda}{\nu q+\theta}} d \tau \\
& \leqslant C \hat{R}^{Q \frac{\nu q-\lambda}{\nu q+\theta}-q \frac{\lambda+\theta}{\nu q+\theta}}\left(\frac{Z(t)^{2 q}}{a(Z(t))}\right)^{\frac{\lambda+\theta}{\nu q+\theta}} \\
& \quad \times\left(\int_{0}^{t} \int_{\hat{R}_{i+1} \leqslant \rho} a(\rho(z)) f(\tau)\left|D_{L} \omega\right|^{q} d z d \tau\right)^{\frac{\lambda+\theta}{\nu q+\theta}} \frac{t^{\frac{\nu q-\lambda}{\nu q+\theta}}}{f(t)^{\frac{\lambda+\theta}{\nu q+\theta}}} .
\end{aligned}
$$


Здесь опять появляется требование к $\theta$. Мы выбрали его достаточно малым, чтобы $-\mu \frac{\lambda+\theta}{\nu q-\lambda}+1>0$. Введем обозначение

$$
\begin{gathered}
y^{(i)}(\hat{R}) \equiv \sup _{0<\tau<t} \int_{\rho \geqslant \hat{R}_{i}} u^{\theta+1} d z+\int_{0}^{t} \int_{\rho \geqslant \hat{R}_{i}} u^{\theta-1}\left|D_{L} u\right|^{\lambda+1} d z d \tau \\
+\int_{0}^{t} \int_{\rho \geqslant \hat{R}_{i}} a(\rho(z)) f(\tau) u^{\theta}\left|D_{L} u^{\nu}\right|^{q} d z d \tau+\frac{2^{i(\lambda+1)}}{R^{\lambda+1}} \int_{0}^{t} \int_{\hat{R}_{i+1} \leqslant \rho \leqslant \hat{R}_{i}} u^{\lambda+\theta} d z d \tau, \\
\varepsilon=\frac{\nu q-\lambda}{\nu q+\theta} .
\end{gathered}
$$

Аналогично лемме 3.3 получим оценку

$$
\begin{aligned}
y^{(i)}(\hat{R}) & \leqslant C \frac{2^{i(\lambda+1)}}{R^{\lambda+1}} R^{Q \frac{\nu q-\lambda}{\nu q+\theta}-q \frac{\lambda+\theta}{\nu q+\theta}} \frac{t^{\frac{\nu q-\lambda}{\nu q+\theta}}}{f(t)^{\frac{\lambda+\theta}{\nu q+\theta}}}\left(\frac{Z(t)^{2 q}}{a(Z(t))}\right)^{\frac{\lambda+\theta}{\nu q+\theta}} y^{(i+1)}(\hat{R})^{\frac{\lambda+\theta}{\nu q+\theta}} \\
& =C 2^{i(\lambda+1)} B\left[y^{(i+1)}(\hat{R})\right]^{1-\varepsilon} .
\end{aligned}
$$

Имея оценки (2.11)-(2.13) и (3.23) можем утверждать, что для $\tau \in(0, t)$

$$
y^{(i)}(\hat{R}) \leqslant C_{1}^{\prime}\left(\hat{R}, t,\left\|u_{0}\right\|_{1, \mathbb{R}^{N+M}}\right) \quad \text { сразу для всех } i,
$$

откуда по итерационной лемме (предложение 6) имеем

$$
\left[y^{(0)}(\hat{R})\right]^{\varepsilon} \leqslant C B .
$$

Оценим с помощью полученного неравенства $y_{0}$ из условия (3.20)

$$
\begin{aligned}
y_{0} \equiv & \sup _{0<\tau<t} \int_{\tilde{U}_{0}} u^{\theta+1} d z+\int_{0}^{t} \int_{\tilde{U}_{0}} u^{\theta-1}\left|D_{L} u\right|^{\lambda+1} d z d \tau \\
& +\int_{0}^{t} \int_{\tilde{U}_{0}} a(\rho(z)) f(\tau) u^{\theta}\left|D_{L} u^{\nu}\right|^{q} d z d \tau+\frac{2^{i(\lambda+1)}}{R^{\lambda+1}} \int_{0}^{t} \int_{U_{0} \backslash \tilde{U}_{0}} u^{\lambda+\theta} d z d \tau \\
\leqslant & y^{(0)}\left(\frac{5 R}{4}\right)+y^{(0)}(R) \leqslant C B^{\frac{1}{\varepsilon}}=C B^{\frac{\nu q+\theta}{\nu q-\lambda}} .
\end{aligned}
$$

Радиусы подобраны с учетом того, что $\tilde{R}_{0}=5 R / 8, R_{0}=R / 2, \hat{R}_{0}=\hat{R} / 2$, $\hat{R}_{1}=3 \hat{R} / 8$. Для доказательства леммы требуется найти условие, при котором справедливо (3.20). С учетом полученной оценки нам потребуется доказать, что

$$
\left(B^{\frac{\nu q+\theta}{\nu q-\lambda}} B^{b}\right)^{\frac{1-a}{b}} A^{a} \leqslant \delta .
$$

Используя второй раз обозначения (3.21) и возведя неравенство в степень

$$
\frac{\left[(\nu q-\lambda)\left(K_{1+\theta}+\lambda^{2}-1\right)\right]}{[(\lambda+1)(\lambda+\theta)]},
$$

получим условие в виде

$$
\left(\frac{Z(t)^{2 q}}{a(Z(t))}\right)^{\lambda-1} R^{(\lambda+1)(1-\nu q)-q(\lambda-1)} \frac{t^{\nu q-\lambda}}{f(t)^{\lambda-1}} \leqslant \delta .
$$

Доказательство леммы завершено, поскольку $(\lambda+1)(1-\nu q)=-q(\lambda-1)-H$. 
Если выбрать $R$ достаточно большим, чтобы выполнялось равенство в условии (3.24), по итерационной лемме (предложение 5) $y_{i} \rightarrow 0$ при $i \rightarrow \infty$. Откуда следует, что $u=0$ п.в. вне $B_{R}, Z(t) \leqslant R$. Значит, из условия (3.24) следует

$$
\frac{t^{\nu q-\lambda}}{f(t)^{\lambda-1}} \leqslant \delta[a(R)]^{\lambda-1} R^{H}
$$

и справедливо

$$
Z(t) \leqslant \varphi\left(\frac{t^{\nu q-\lambda}}{f(t)^{\lambda-1}}\right)
$$

По определению (1.6) ясно, что верно (1.10). Доказательство теоремы 1 завершено.

\section{§ 4. Доказательство теоремы 2}

Докажем оценку (1.11). Умножим обе части уравнения (1.1) на $u^{\theta}, \theta>0$, и проинтегрируем на шаре $B_{Z(t)}$. Для $0<\tau<t$ имеем

$$
\begin{gathered}
\frac{1}{1+\theta} \frac{d}{d \tau} \int_{B_{Z(t)}} u^{\theta+1} d z \leqslant-\int_{B_{Z(t)}} a(\rho(z)) f(\tau) u^{\theta}\left|D_{L} u^{\nu}\right|^{q} d z \\
\leqslant-C \int_{B_{Z(t)}} a(\rho(z)) f(\tau)\left|D_{L} u^{\frac{\nu q+\theta}{q}}\right|^{q} d z .
\end{gathered}
$$

Рассмотрим два интеграла. Первый:

$$
\int_{B_{Z(t)}} u^{\theta+1} d z \leqslant\left(\int_{B_{Z(t)}} u^{\nu q+\theta}\left|D_{L} \rho\right|^{q} d z\right)^{\frac{1+\theta}{\nu q+\theta}}\left(\int_{B_{Z(t)}}\left|D_{L} \rho\right|^{-q \frac{1+\theta}{\nu q-1}} d z\right)^{\frac{\nu q-1}{\nu q+\theta}} .
$$

Используем замену переменных из предложения 4. Множество

$$
B_{R} \equiv\left\{\begin{array}{c}
0 \leqslant \rho \leqslant R \\
0 \leqslant \varphi_{1} \leqslant 2 \pi \\
0 \leqslant \varphi_{2}<\pi \\
\cdots \\
0 \leqslant \varphi_{M+N-1}<\pi
\end{array}\right\}
$$

обозначим

$$
\Psi \equiv\left\{\begin{array}{c}
0 \leqslant \varphi_{1} \leqslant 2 \pi \\
0 \leqslant \varphi_{2}<\pi \\
\cdots \\
0 \leqslant \varphi_{M+N-1}<\pi
\end{array}\right\}
$$

и мы получим оценку второго множителя:

$$
\begin{aligned}
& \int_{B_{R}}\left|D_{L} \rho\right|^{-q \frac{1+\theta}{\nu q-1}} d z=\int_{B_{R}}\left(\frac{\rho^{\alpha}}{|x|^{\alpha}}\right)^{q \frac{1+\theta}{\nu q-1}} J(\rho, \varphi) d \rho d \varphi_{1} \cdots d \varphi_{M+N-1} \\
& =\int \frac{J(\rho, \varphi) d \rho d \varphi}{\left(\left(\cos \varphi_{M+N-1} \cdots \cos \varphi_{1}\right)^{2}+\cdots+\left(\cos \varphi_{M+N-1} \cdots \sin \varphi_{M-1}\right)^{2}\right)^{\frac{\alpha q(\theta+1)}{2(\alpha+1)(\nu q-1)}}} \\
& =\int_{0}^{R} \rho^{Q-1} d \rho \int \cdots \int_{\Psi} \Phi(\varphi) d \varphi_{1} \cdots d \varphi_{M+N-1} \leqslant C R^{Q},
\end{aligned}
$$


где

$$
\begin{aligned}
& \Phi(\varphi)= \frac{\cos ^{\frac{1}{\alpha+1}} \varphi_{2} \cos ^{\frac{2}{\alpha+1}} \varphi_{3} \cdots \cos ^{\frac{M-2}{\alpha+1}} \varphi_{M-1} \cos ^{\frac{M-1}{\alpha+1}} \varphi_{M} \cos ^{\frac{M}{\alpha+1}} \varphi_{M+1}}{\left(\cos \varphi_{M+N-1} \cdots \varphi_{M+1}\right)^{\frac{\alpha q(1+\theta)}{(\alpha+1)(\nu q-1)}}\left(\sin \varphi_{1} \cdots \sin \varphi_{M-1}\right)^{\frac{\alpha}{\alpha+1}}} \\
& \times \frac{\cos ^{\frac{M}{\alpha+1}+1} \varphi_{M+2} \cdots \cos ^{\frac{M}{\alpha+1}}+N-2}{\varphi_{M+N-1}} \\
& \cos \varphi_{M}^{\frac{\alpha q(1+\theta)}{(\alpha+1)(\nu q-1)}+\frac{\alpha}{\alpha+1}}\left(\cos \varphi_{1} \cdots \cos \varphi_{M-1}\right)^{\frac{\alpha}{\alpha+1}}
\end{aligned}
$$

Действительно, интеграл по $d \varphi$ может иметь особенности только для $\varphi_{j}=\varphi_{01 j}$, поскольку $\sin \varphi_{01 j}=0, j=\overline{M-1, M+N-1}$, и $\varphi_{j}=\varphi_{02 j}$, поскольку $\cos \varphi_{02 j}=0$, $j=\overline{M, M+N-1}$. Рассмотрим эти случаи. В окрестности точки $\varphi_{01 j}$ функция под интегралом $\Phi(\varphi)$ будет эквивалентна

$$
\frac{1}{\sin \left(\varphi_{j}-\varphi_{01 j}\right)^{\frac{\alpha}{\alpha+1}}} \sim \frac{1}{\left(\varphi_{j}-\varphi_{01 j}\right)^{\frac{\alpha}{\alpha+1}}}
$$

т.е. имеет устранимую особенность. В окрестности точки $\varphi_{02}$ м функция под интегралом $\Phi(\varphi)$ будет эквивалентна $\left(\varphi_{M}-\varphi_{02 M}\right)^{\frac{M-1}{\alpha+1}-\frac{\alpha q(\theta+1)}{(\alpha+1)(\nu q-1)}-\frac{\alpha}{\alpha+1}}$, особенность устранима, если

$$
M-\frac{\alpha q}{\nu q-1}>0, \quad 0<\theta \leqslant \frac{M(\nu q-1)-\alpha q}{\alpha q} .
$$

В окрестности точки $\varphi_{02 j}, j=\overline{M+1, M+N-1}$, функция под интегралом

$$
\Phi(\varphi) \sim\left(\varphi_{j}-\varphi_{02 j}\right)^{\frac{M}{\alpha+1}+(j-M-1)-\frac{\alpha q(\theta+1)}{(\alpha+1)(\nu q-1)}},
$$

особенность устранима, если условие

$$
M-\frac{\alpha q}{\nu q-1}>0, \quad 0<\theta \leqslant \frac{M(\nu q-1)-\alpha q}{\alpha q}
$$

в силе. В окрестности точки $\varphi_{02 j}, j=\overline{1, M-1}$, функция под интегралом $\Phi(\varphi) \sim\left(\varphi_{j}-\varphi_{02 j}\right)^{\frac{j-1}{\alpha+1}-\frac{\alpha}{\alpha+1}}$, особенность устранима. Значит, интеграл по $d \varphi$ сходится к некоторой постоянной величине. Имеем теперь

$$
\int_{B_{Z(t)}} u^{\theta+1} d z \leqslant C\left(\int_{B_{Z(t)}} u^{\nu q+\theta}\left|D_{L} \rho\right|^{q} d z\right)^{\frac{1+\theta}{\nu q+\theta}} Z(t)^{Q \frac{\nu q-1}{\nu q+\theta}} .
$$

Рассмотрим второй интеграл.

$$
\begin{aligned}
& -C \int_{B_{Z(t)}} a(\rho(z)) f(\tau)\left|D_{L} u^{\frac{\nu q+\theta}{q}}\right|^{q} d z \leqslant-C \int_{B_{Z(t)}} \frac{a(\rho(z))}{\rho^{q}} f(\tau)\left|D_{L} \rho\right|^{q}\left|u^{\frac{\nu q+\theta}{q}}\right|^{q} d z \\
& \quad \leqslant-C Z(t)^{-q} a(Z(t)) f(\tau) \int_{B_{Z(t)}}\left|D_{L} \rho\right|^{q}\left|u^{\frac{\nu q+\theta}{q}}\right|^{q} d z \\
& \quad=-C Z(t)^{-q} a(Z(t)) f(\tau) \int_{B_{Z(t)}}\left|D_{L} \rho\right|^{q} u^{\nu q+\theta} d z .
\end{aligned}
$$

Здесь мы использовали неравенство типа Харди (2.3). Следовательно,

$$
\frac{d}{d \tau}\left(\int_{B_{Z(t)}} u^{\theta+1} d z\right) \leqslant-C Z(t)^{-q} a(Z(t)) f(\tau) Z(t)^{-\frac{Q(\nu q-1)}{1+\theta}}\left(\int_{B_{Z(t)}} u^{\theta+1} d z\right)^{\frac{\nu q+\theta}{1+\theta}} .
$$


Решив это дифференциальное неравенство, получим

$$
\int_{B_{Z(t)}} u^{\theta+1} d z \leqslant C Z(t)^{\frac{q(1+\theta)}{\nu q-1}+Q} a(Z(t))^{-\frac{1+\theta}{\nu q-1}} t^{-\frac{1+\theta}{\nu q-1}} f(t)^{-\frac{1+\theta}{\nu q-1}} .
$$

Оценим массу решения

$$
\begin{aligned}
\int_{\mathbb{R}^{N+M}} u d z & =\int_{B_{Z(t)}} u d z \leqslant\left(\int_{B_{Z(t)}} u^{\theta+1} d z\right)^{\frac{1}{1+\theta}} \operatorname{mes}^{\frac{\theta}{1+\theta}}\left\{B_{Z(t)}\right\} \\
& \leqslant C \frac{Z(t)^{Q+\frac{q}{\nu q-1}}}{a(Z(t))^{\frac{1}{\nu q-1}} t^{\frac{1}{\nu q-1}} f(t)^{\frac{1}{\nu q-1}}} .
\end{aligned}
$$

По определению (1.6) ясно, что

$$
\begin{gathered}
\omega(t) t^{\frac{1}{K}}=\varphi\left(\frac{t^{\nu q-\lambda}}{f(t)^{\lambda-1}}\right), \quad a\left(\omega(t) t^{\frac{1}{K}}\right)^{\lambda-1}\left(\omega(t) t^{\frac{1}{K}}\right)^{H}=\frac{t^{\nu q-\lambda}}{f(t)^{\lambda-1}}, \\
a\left(\omega(t) t^{\frac{1}{K}}\right) f(t)=\frac{t^{\frac{\nu q-\lambda}{\lambda-1}-\frac{H}{K(\lambda-1)}}}{\omega^{\frac{H}{\lambda-1}}(t)} .
\end{gathered}
$$

Используя (4.4), из неравенства (4.5) легко получить (1.11). Получим соответствующую оценку $L_{\infty}$-нормы решения. Известно, следуя предложению 8 , что для $t>t_{0}=R_{0}^{-K}\left\|u_{0}\right\|_{1, \mathbb{R}^{N+M}}^{\lambda-1}$

$$
\begin{gathered}
\|u\|_{\infty, \mathbb{R}^{N+M}} \leqslant C\|u\|_{1, \mathbb{R}^{N+M}}^{\frac{\lambda+1}{K}} t^{-\frac{Q}{K}} \leqslant C\|u\|_{\infty, \mathbb{R}^{N+M}}^{\frac{\lambda+1}{K}} Z(t)^{\frac{Q(\lambda+1)}{K}} t^{-\frac{Q}{K}}, \\
\|u\|_{\infty, \mathbb{R}^{N+M}}^{1-\frac{\lambda+1}{K}} \leqslant C Z(t)^{\frac{\lambda+1}{\lambda-1}} t^{-\frac{1}{\lambda-1}} .
\end{gathered}
$$

Теорема 2 доказана.

\section{§5. Доказательство теоремы 3}

В этой теореме исследуются два случая $\lambda=1$ и $\nu=1$, где можно получить оценку массы решения без использования компактности носителя начальной функции.

$$
\int_{\mathbb{R}^{N+M}} u d z=\int_{B_{R}} u d z+\int_{\mathbb{R}^{N+M} \backslash B_{R}} u d z:=E_{1}(t)+E_{2}(t) .
$$

Здесь $R$ - фиксированное число, которое будет выбрано позднее.

$$
\begin{aligned}
E_{1}(t) & =\int_{B_{R}} u d z \leqslant\left(\int_{B_{R}} u^{\nu q}\left|D_{L} \rho\right|^{q} d z\right)^{\frac{1}{\nu q}}\left(\int_{B_{R}}\left|D_{L} \rho\right|^{-q \frac{1}{\nu q-1}} d z\right)^{\frac{\nu q-1}{\nu q}} \\
& =I_{1}^{\frac{1}{\nu q}} I_{2}^{\frac{\nu q-1}{\nu q}} .
\end{aligned}
$$

Используем предложение 3:

$$
I_{1} \leqslant \frac{R^{q}}{a(R)} \int_{B_{R}} \frac{u^{\nu q}\left|D_{L} \rho\right|^{q} a(\rho)}{\rho^{q}} d z \leqslant C \frac{R^{q}}{a(R)} \int_{\mathbb{R}^{N+M}} a(\rho(z))\left|D_{L} u^{\nu}\right|^{q} d z .
$$


Рассмотрим второй множитель, использовав замену переменных (2.5) из предложения 4. Мы получим

$$
\begin{aligned}
I_{2} & =\int_{B_{R}}\left|D_{L} \rho\right|^{-q \frac{1}{\nu q-1}} d z=\int_{B_{R}}\left(\frac{\rho^{\alpha}}{|x|^{\alpha}}\right)^{q \frac{1}{\nu q-1}} J(\rho, \varphi) d \rho d \varphi_{1} \cdots d \varphi_{M+N-1} \\
& =\int \frac{J(\rho, \varphi) d \rho d \varphi}{\left(\left(\cos \varphi_{M+N-1} \cdots \cos \varphi_{1}\right)^{2}+\cdots+\left(\cos \varphi_{M+N-1} \cdots \sin \varphi_{M-1}\right)^{2}\right)^{\frac{\alpha q}{2(\alpha+1)(\nu q-1)}}} \\
& =\int_{0}^{R} \rho^{Q-1} d \rho \iint_{\Psi} \Phi(\varphi) d \varphi_{1} \cdots d \varphi_{M+N-1} \leqslant C R^{Q},
\end{aligned}
$$

где

$$
\begin{gathered}
\Phi(\varphi)=\frac{\cos ^{\frac{1}{\alpha+1}} \varphi_{2} \cos ^{\frac{2}{\alpha+1}} \varphi_{3} \cdots \cos ^{\frac{M-2}{\alpha+1}} \varphi_{M-1} \cos ^{\frac{M-1}{\alpha+1}} \varphi_{M} \cos ^{\frac{M}{\alpha+1}} \varphi_{M+1}}{\left(\cos \varphi_{M+N-1} \cdots \cos \varphi_{M+1}\right)^{\frac{\alpha q}{(\alpha+1)(\nu q-1)}}\left(\sin \varphi_{1} \cdots \sin \varphi_{M-1}\right)^{\frac{\alpha}{\alpha+1}}} \\
\quad \times \frac{\cos ^{\frac{M}{\alpha+1}+1} \varphi_{M+2} \cdots \cos ^{\frac{M}{\alpha+1}}+N-2}{\varphi_{M+N-1}} \\
\cos \varphi_{M} \frac{\alpha q}{(\alpha+1)(\nu q-1)}+\frac{\alpha}{\alpha+1}\left(\cos \varphi_{1} \cdots \cos \varphi_{M-1}\right)^{\frac{\alpha}{\alpha+1}}
\end{gathered}
$$

Действительно, интеграл по $d \varphi$ может иметь особенности только для $\varphi_{j}=$ $\varphi_{01 j}$, поскольку $\sin \varphi_{01 j}=0, j=\overline{M-1, M+N-1}$, и $\varphi_{j}=\varphi_{02 j}$, поскольку $\cos \varphi_{02 j}=0, j=\overline{M, M+N-1}$. Рассмотрим эти случаи. В окрестности точки $\varphi_{01 j}$ функция под интегралом $\Phi(\varphi)$ будет эквивалентна

$$
\frac{1}{\sin \left(\varphi_{j}-\varphi_{01 j}\right)^{\frac{\alpha}{\alpha+1}}} \sim \frac{1}{\left(\varphi_{j}-\varphi_{01 j}\right)^{\frac{\alpha}{\alpha+1}}}
$$

т.е. имеет устранимую особенность. В окрестности точки $\varphi_{02}$ м функция под интегралом $\Phi(\varphi)$ будет эквивалентна $\left(\varphi_{M}-\varphi_{02 M}\right)^{\frac{M-1}{\alpha+1}-\frac{\alpha q}{(\alpha+1)(\nu q-1)}-\frac{\alpha}{\alpha+1}}$, особенность устранима, если $M-\alpha q /(\nu q-1)>0$. В окрестности точки $\varphi_{02 j}, j=$ $\overline{M+1, M+N-1}$, функция под интегралом

$$
\Phi(\varphi) \sim\left(\varphi_{j}-\varphi_{02 j}\right)^{\frac{M}{\alpha+1}+(j-M-1)-\frac{\alpha q}{(\alpha+1)(\nu q-1)}},
$$

особенность устранима, если условие $M-\alpha q /(\nu q-1)>0$ в силе. В окрестности точки $\varphi_{02 j}, j=\overline{1, M-1}$, функция под интегралом $\Phi(\varphi) \sim\left(\varphi_{j}-\varphi_{02 j}\right)^{\frac{j-1}{\alpha+1}-\frac{\alpha}{\alpha+1}}$, особенность устранима. Значит, интеграл по $d \varphi$ сходится к некоторой постоянной величине. В итоге имеем

$$
E_{1}(t) \leqslant C\left(\frac{R^{q}}{a(R) f(t)} \int_{\mathbb{R}^{N+M}} a(\rho(z)) f(t)\left|D_{L} u^{\nu}\right|^{q} d z\right)^{\frac{1}{\nu q}} R^{Q \frac{\nu q-1}{\nu q}} .
$$

Если проинтегрировать уравнение по $\mathbb{R}^{N+M}$ и перебросить производные во втором слагаемом, получим

$$
\frac{d}{d \tau} \int_{\mathbb{R}^{N+M}} u d z=-\int_{\mathbb{R}^{N+M}} a(\rho(z)) f(\tau)\left|D_{L} u^{\nu}\right|^{q} d z .
$$

Тогда имеем

$$
\int_{\mathbb{R}^{N+M}} u(\tau) d z \leqslant C \frac{R^{\frac{q}{\nu q}+Q \frac{\nu q-1}{\nu q}}}{f(\tau)^{\frac{1}{\nu q}} a(R)^{\frac{1}{\nu q}}}\left(-\frac{d}{d \tau} \int_{\mathbb{R}^{N+M}} u(\tau) d z\right)^{\frac{1}{\nu q}}+E_{2}(\tau) .
$$


Оценим $E_{2}(\tau)$ для случая $\nu=1$. Уравнение примет вид

$$
\begin{gathered}
u_{t}=\operatorname{div}_{L}\left(\left|D_{L} u\right|^{\lambda-1} D_{L} u\right)-a(\rho(z)) f(t)\left|D_{L} u\right|^{q}, \\
(z, t) \in S_{T}=\mathbb{R}^{N+M} \times\left(0, T_{r}\right), \quad \lambda<q<\lambda+1 .
\end{gathered}
$$

Выберем срезающую функцию

$$
\xi(z)=\left\{\begin{array}{ll}
0 & \text { при } \rho(z)<\frac{R}{2}, \\
1 & \text { при } \rho(z)>R,
\end{array} \quad 0 \leqslant \xi \leqslant 1, \quad\left|D_{L} \xi\right| \leqslant \frac{C}{R} .\right.
$$

Умножим уравнение на $\xi^{s}$, где $s=q /(q-\lambda)>\lambda+1$, и проинтегрируем по частям на множестве $\mathbb{R}^{N+M}$. Затем получим неравенство

$$
\frac{d}{d \tau} \int_{\mathbb{R}^{N+M}} u \xi^{s} d z+\int_{\mathbb{R}^{N+M}} a(\rho(z)) f(\tau)\left|D_{L} u\right|^{q} \xi^{s} d z \leqslant \frac{C}{R} \int_{\frac{R}{2} \leqslant \rho \leqslant R}\left|D_{L} u\right|^{\lambda} \xi^{s-1} d z .
$$

Оценим правую часть, использовав неравенство Гёльдера с показателем $p=$ $q / \lambda=s /(s-1)$ и неравенство Юнга.

$$
\begin{aligned}
& \frac{C}{R} \int_{\frac{R}{2} \leqslant \rho \leqslant R}\left|D_{L} u\right|^{\lambda} \xi^{s-1} d z \\
& \quad \leqslant \frac{C}{R}\left(\int_{\frac{R}{2} \leqslant \rho \leqslant R} a(\rho) f(\tau)\left|D_{L} u\right|^{q} \xi^{s} d z\right)^{\frac{q}{\lambda}}\left(\int_{\frac{R}{2} \leqslant \rho \leqslant R}[a(\rho) f(\tau)]^{-\frac{\lambda}{q-\lambda}} d z\right)^{\frac{q-\lambda}{q}} \\
& \quad \leqslant \frac{1}{2} \int_{\mathbb{R}^{N+M}} a(\rho(z)) f(\tau)\left|D_{L} u\right|^{q} \xi^{s} d z+\frac{C}{R^{\frac{q}{q-\lambda}}} \int_{\frac{R}{2} \leqslant \rho \leqslant R}[a(\rho) f(\tau)]^{-\frac{\lambda}{q-\lambda}} d z
\end{aligned}
$$

Получаем дифференциальное неравенство

$$
\frac{d}{d \tau} \int_{\mathbb{R}^{N+M}} u \xi^{s} d z \leqslant C \frac{R^{Q-\frac{q}{q-\lambda}}}{a(R)^{\frac{\lambda}{q-\lambda}} f(\tau)^{\frac{\lambda}{q-\lambda}}} .
$$

Проинтегрируем его на множестве $(0, \tau)$.

$$
\begin{aligned}
& \int_{\mathbb{R}^{N+M}} u(z, \tau) \xi^{s} d z \leqslant \int_{\mathbb{R}^{N+M}} u_{0} \xi^{s} d z+C \frac{R^{Q-\frac{q}{q-\lambda}}}{a(R)^{\frac{\lambda}{q-\lambda}}} \int_{0}^{\tau} f(\eta)^{-\frac{\lambda}{q-\lambda}} d \eta \\
& \quad \leqslant \int_{\mathbb{R}^{N+M}} u_{0} \xi^{s} d z+C \frac{\tau R^{Q-\frac{q}{q-\lambda}}}{a(R)^{\frac{\lambda}{q-\lambda}} f(\tau)^{\frac{\lambda}{q-\lambda}}} \equiv \Upsilon(R, \tau) \leqslant \Upsilon(R, t), \quad 0<t_{1}<\tau<t .
\end{aligned}
$$

Значит, получаем

$$
E_{2}(R, \tau) \leqslant \Upsilon(R, t) .
$$

Объединяя оценки $E_{1}, E_{2}$, имеем

$$
E(\tau) \leqslant \Upsilon(R, t)+C \frac{R^{\frac{q}{\nu q}+Q \frac{\nu q-1}{\nu q}}}{f(\tau)^{\frac{1}{\nu q}} a(R)^{\frac{1}{\nu q}}}\left(-\frac{d}{d \tau} E(\tau)\right)^{\frac{1}{\nu q}} .
$$

Оценим $E_{2}(\tau)$ для случая $\lambda=1$. Уравнение примет вид

$$
\begin{aligned}
u_{t} & =\operatorname{div}_{L}\left(D_{L} u\right)-a(\rho(z)) f(t)\left|D_{L} u^{\nu}\right|^{q}, \\
(z, t) \in S_{T} & =\mathbb{R}^{N+M} \times\left(0, T_{r}\right), \quad 1<q<2, \quad \nu q>1 .
\end{aligned}
$$


Выберем срезающую функцию

$$
\xi(z)=\left\{\begin{array}{ll}
0 & \text { при } \rho(z)<R, \\
1 & \text { при } \rho(z)>2 R,
\end{array} \quad 0 \leqslant \xi \leqslant 1, \quad\left|D_{L} \xi\right| \leqslant \frac{C}{R}, \quad\left|\operatorname{div}_{L}\left(D_{L} \xi\right)\right| \leqslant \frac{C}{R^{2}} .\right.
$$

Умножим уравнение на $\xi^{2}$ и проинтегрируем по частям на множестве $\mathbb{R}^{N+M} \times$ $(0, \tau)$. Получим

$$
\begin{gathered}
\int_{\mathbb{R}^{N+M}} u(\tau) \xi^{2} d z+\int_{0}^{\tau} \int_{\mathbb{R}^{N+M}} a(\rho(z)) f(\eta)\left|D_{L} u^{\nu}\right|^{q} \xi^{2} d z d \eta \\
\quad=\int_{\mathbb{R}^{N+M}} u_{0} \xi^{2} d z+\int_{0}^{\tau} \int_{\mathbb{R}^{N+M}} u \cdot \operatorname{div}_{L}\left(D_{L} \xi^{2}\right) d z d \eta \\
\leqslant \int_{\rho>R} u_{0} d z+\frac{C}{R} \int_{0}^{\tau} \int_{2 R>\rho>R} u d z d \eta
\end{gathered}
$$

Используя (5.1), получим оценку

$$
\begin{aligned}
& \frac{C}{R^{2}} \int_{0}^{\tau} \int_{2 R>\rho} u d z d \eta \\
& \quad \leqslant \frac{C}{R^{2}} \int_{0}^{\tau}\left(\frac{R^{q}}{a(R) f(\eta)} \int_{\mathbb{R}^{N+M}} a(\rho(z)) f(\eta)\left|D_{L} u^{\nu}\right|^{q} d z\right)^{\frac{1}{\nu q}} R^{Q \frac{\nu q-1}{\nu q}} d \eta \\
& \quad \leqslant C \frac{R^{\frac{Q(\nu q-1)+q-2 \nu q}{\nu q}} \tau^{\frac{\nu q-1}{\nu q}}}{a(R)^{\frac{1}{\nu q}} f(\tau)^{\frac{1}{\nu q}}}\left(\int_{0}^{\tau} \int_{\mathbb{R}^{N+M}} a(\rho(z)) f(\eta)\left|D_{L} u^{\nu}\right|^{q} d z d \eta\right)^{\frac{1}{\nu q}} .
\end{aligned}
$$

Применим неравенство Юнга, чтобы получить

$$
\begin{aligned}
\int_{\rho>2 R} u(\tau) d z \leqslant \int_{\rho>R} u_{0} d z & \\
& +C \frac{R^{\frac{Q(\nu q-1)+q-2 \nu q}{\nu q}} \tau^{\frac{\nu q-1}{\nu q}}}{a(R)^{\frac{1}{\nu q}} f(\tau)^{\frac{1}{\nu q}}}\left(\int_{0}^{\tau} \int_{\mathbb{R}^{N+M}} a(\rho(z)) f(\eta)\left|D_{L} u^{\nu}\right|^{q} d z d \eta\right)^{\frac{1}{\nu q}} \\
\leqslant & \int_{\rho>R} u_{0} d z+\frac{1}{2} \int_{0}^{\tau} \int_{\mathbb{R}^{N+M}} a(\rho(z)) f(\eta)\left|D_{L} u^{\nu}\right|^{q} d z d \eta+C \frac{R^{\frac{Q(\nu q-1)+q-2 \nu q}{\nu q-1}} \tau}{a(R)^{\frac{1}{\nu q-1}} f(\tau)^{\frac{1}{\nu q-1}}} \\
\leqslant & \int_{\rho>R} u_{0} d z+\frac{1}{2}\left(\int_{\mathbb{R}^{N+M}} u(\tau) d z-\int_{\mathbb{R}^{N+M}} u_{0} d z\right)+C \frac{R^{\frac{Q(\nu q-1)+q-2 \nu q}{\nu q-1}} \tau}{a(R)^{\frac{1}{\nu q-1}} f(\tau)^{\frac{1}{\nu q-1}}} .
\end{aligned}
$$

Если использовать обозначения, имеем

$$
E_{2}(\tau) \leqslant \frac{1}{2} \int_{\rho>R} u_{0} d z+\frac{1}{2} E(\tau)+C \frac{R^{\frac{Q(\nu q-1)+q-2 \nu q}{\nu q-1}} \tau}{a(R)^{\frac{1}{\nu q-1}} f(\tau)^{\frac{1}{\nu q-1}}} .
$$

Аналогично предыдущему случаю обозначим

$$
\Upsilon(R, \tau) \equiv \frac{1}{2} \int_{\rho>R} u_{0} d z+C \frac{R^{\frac{Q(\nu q-1)+q-2 \nu q}{\nu q-1}} \tau}{a(R)^{\frac{1}{\nu q-1}} f(\tau)^{\frac{1}{\nu q-1}}} \leqslant \Upsilon(R, t), \quad 0<t_{1}<\tau<t .
$$

Объединяя оценки $E_{1}, E_{2}$, получим

$$
E(\tau) \leqslant \frac{1}{2} E(\tau)+\Upsilon(R, t)+C \frac{R^{\frac{q}{\nu q}+Q \frac{\nu q-1}{\nu q}}}{f(\tau)^{\frac{1}{\nu q}} a(R)^{\frac{1}{\nu q}}}\left(-\frac{d}{d \tau} E(\tau)\right)^{\frac{1}{\nu q}} .
$$


В обоих случаях $(\lambda=1$ и $\nu=1)$ справедлива оценка (5.5). Переобозначим в ней $y(\tau)=E(\tau)-\Upsilon(R, t)$. Полученное проинтегрируем от 0 до $t$ и решим

$$
y(\tau) \leqslant C \frac{R^{\frac{q}{\nu q}+Q \frac{\nu q-1}{\nu q}}}{f(\tau)^{\frac{1}{\nu q}} a(R)^{\frac{1}{\nu q}}}\left(-y_{\tau}(\tau)\right)^{\frac{1}{\nu q}}, \quad y(t) \leqslant C \frac{R^{Q+\frac{q}{\nu q-1}}}{a(R)^{\frac{1}{\nu q-1}} t^{\frac{1}{\nu q-1}} f(t)^{\frac{1}{\nu q-1}} .} .
$$

Отсюда для случая $\nu=1$ имеем

$$
\int_{\mathbb{R}^{N+M}} u(t) d z \leqslant \int_{\rho>R} u_{0} d z+C \frac{t R^{Q-\frac{q}{q-\lambda}}}{a(R)^{\frac{\lambda}{q-\lambda}} f(t)^{\frac{\lambda}{q-\lambda}}}+C \frac{R^{Q+\frac{q}{q-1}}}{a(R)^{\frac{1}{q-1}} t^{\frac{1}{q-1}} f(t)^{\frac{1}{q-1}}} .
$$

Последние два слагаемых совпадают с точностью до константы, если

$$
R=R(t): R^{H} a(R)^{\lambda-1}=\frac{t^{q-\lambda}}{f(t)^{\lambda-1}} .
$$

Доказательство неравенства (1.13) для случая $\nu=1$ закончено. Для случая, когда $\lambda=1$, получается

$$
\int_{\mathbb{R}^{N+M}} u(t) d z \leqslant \int_{\rho>R} u_{0} d z+C \frac{R^{\frac{Q(\nu q-1)+q-2 \nu q}{\nu q-1}} t}{a(R)^{\frac{1}{\nu q-1}} f(t)^{\frac{1}{\nu q-1}}}+C \frac{R^{Q+\frac{q}{\nu q-1}}}{a(R)^{\frac{1}{\nu q-1}} t^{\frac{1}{\nu q-1}} f(t)^{\frac{1}{\nu q-1}}} .
$$

Последние два слагаемых совпадают с точностью до константы, если $R=\sqrt{t}$. Доказательство теоремы 3 закончено.

\section{§6. Доказательство теоремы 4}

Проинтегрируем (1.1) по шару $B_{Z(t)}$. Имеем

$$
\frac{d}{d t} \int_{B_{Z(t)}} u d z=-\int_{B_{Z(t)}} a(\rho(z)) f(t)\left|D_{L} u^{\nu}\right|^{q} d z .
$$

Используя неравенство (5.1), которое справедливо, если $M-\alpha q /(\nu q-1)>0$, имеем

$$
\begin{aligned}
\int_{B_{Z(t)}} u(z, t) d z & \leqslant\left(\int_{B_{Z(t)}} u^{\nu q}\left|D_{L} \rho\right|^{q} d z\right)^{\frac{1}{\nu q}}\left(\int_{B_{Z(t)}}\left|D_{L} \rho\right|^{-q \frac{1}{\nu q-1}} d z\right)^{\frac{\nu q-1}{\nu q}} \\
& \leqslant C Z(t)^{Q \frac{\nu q-1}{\nu q}}\left(\frac{Z(t)^{q}}{a(Z(t)) f(t)} \int_{B_{Z(t)}} a(\rho(z)) f(t)\left|D_{L} u^{\nu}\right|^{q} d z\right)^{\frac{1}{\nu q}} .
\end{aligned}
$$

Объединив полученные оценки, имеем

$$
\frac{d}{d t} \int_{B_{Z(t)}} u d z \leqslant-C\left(\int_{B_{Z(t)}} u d z\right)^{\nu q} a(Z(t)) Z(t)^{-Q(\nu q-1)-q} f(t) .
$$

Обозначим $y(t)=\int_{B_{Z(t)}} u(z, t) d z$, тогда

$$
\frac{d y(t)}{d t} y^{-\nu q}(t) \leqslant-C a(Z(t)) Z(t)^{-Q(\nu q-1)-q} f(t)
$$


Проинтегрировав неравенство на отрезке $\left(t_{0}, t\right)$, получим

$$
\begin{gathered}
y(t) \leqslant C\left(\int_{t_{0}}^{t} \frac{a(Z(\tau)) f(\tau)}{Z(\tau)^{Q(\nu q-1)+q}} d \tau\right)^{-\frac{1}{\nu q-1}}, \\
\int_{B_{Z(t)}} u d z \leqslant C\left(\int_{t_{0}}^{t} \frac{a(Z(\tau)) f(\tau)}{Z(\tau)^{Q(\nu q-1)+q}} d \tau\right)^{-\frac{1}{\nu q-1}} .
\end{gathered}
$$

Под интегралом находится произведение двух функций: $f(\tau)$ и убывающей по $Z$ функции $a(Z) / Z^{Q(\nu q-1)+q}$.

Пусть выполняется оценка (1.8), тогда для достаточно больших $t$ справедлива оценка $Z(t) \leqslant C t^{\frac{1}{K}}$, откуда

$$
\frac{a(Z(\tau)) f(\tau)}{Z(\tau)^{Q(\nu q-1)+q}} \geqslant \frac{a\left(\tau^{\frac{1}{K}}\right) f(\tau)}{\tau^{\frac{Q(\nu q-1)+q}{K}}} .
$$

Используя последнее неравенство, получим,

$$
\begin{gathered}
\int_{t_{0}}^{t} \frac{a(Z(\tau)) f(\tau)}{Z(\tau)^{Q(\nu q-1)+q}} d \tau \geqslant C \int_{t_{0}}^{t} \frac{a\left(\tau^{\frac{1}{K}}\right) f(\tau)}{\tau^{\frac{Q(\nu q-1)+q}{K}}} d \tau, \\
\int_{\mathbb{R}^{N+M}} u d z \leqslant C\left(\int_{t_{0}}^{t} \frac{a\left(\tau^{\frac{1}{K}}\right) f(\tau)}{\tau^{\frac{Q(\nu q-1)+q}{K}}} d \tau\right)^{-\frac{1}{\nu q-1}} .
\end{gathered}
$$

В случае, если существуют $C>0, \varrho \in(0,1)$ такие, что $C \varrho \leqslant \omega(t) \leqslant C$ для достаточно больших $t, t \geqslant t_{0}=\left\|u_{0}\right\|_{1, \mathbb{R}^{N+M}}^{\lambda-1} / R_{0}^{K}$, имеем

$$
\frac{a(Z(\tau)) f(\tau)}{Z(\tau)^{Q(\nu q-1)+q}} \geqslant C \frac{a\left(\tau^{\frac{1}{K}}\right) f(\tau)}{\tau^{\frac{Q(\nu q-1)+q}{K}}}=C \frac{1}{\tau}, \quad \int_{B_{Z(t)}} u d z \leqslant C\left(\int_{t_{0}}^{t} \tau^{-1} d \tau\right)^{-\frac{1}{\nu q-1}} .
$$

Теорема доказана.

\section{§ 7. Доказательство теоремы 5}

Лемма 7.1. Пусть $u$-решение уравнения (1.1). Тогда $u\left(z, t_{0}\right) \neq 0$ для всех $z \in \mathbb{R}^{N+M}$ и для любого конечного $t_{0}>0$.

ДоказАтельство. Допустим, что существует $t_{0}$ такое, что $\left\|u\left(t_{0}\right)\right\|_{1}=0$, $\|u(t)\|_{1}>0$ для $t<t_{0}$. Пусть $\vartheta, 0<\vartheta<1,-$ параметр. Введем обозначение

$$
E_{\vartheta}(t)=\int_{\mathbb{R}^{N+M}} u(t)^{1+\vartheta} d z .
$$

Тогда интегрирование по частям уравнения по всему $\mathbb{R}^{N+M}$ дает $(5.2)$, оценим интеграл справа, используя неравенство Гёльдера

$$
\begin{aligned}
\int_{\mathbb{R}^{N+M}} a(\rho(z)) f(\tau)\left|D_{L} u^{\nu}\right|^{q} d z=\int_{\mathbb{R}^{N+M}} a(\rho(z)) f(t)\left|\nu u^{\nu-1} D_{L} u\right|^{q} u^{\frac{(\vartheta-1) q}{\lambda+1}} u^{-\frac{(\vartheta-1) q}{\lambda+1}} d z \\
\leqslant C\left(\int_{\mathbb{R}^{N+M}} u^{\vartheta-1}\left|D_{L} u\right|^{\lambda+1} d z\right)^{\frac{q}{\lambda+1}} \\
\quad \times\left(\int_{\mathbb{R}^{N+M}} a(\rho(z))^{\frac{\lambda+1}{\lambda+1-q}} f(t)^{\frac{\lambda+1}{\lambda+1-q}}\left[u^{(\nu-1) q-\frac{(\vartheta-1) q}{\lambda+1}}\right]^{\frac{\lambda+1}{\lambda+1-q}} d z\right)^{\frac{\lambda+1-q}{\lambda+1}} \\
=C H_{1}^{\frac{q}{\lambda+1}} H_{2}^{\frac{\lambda+1-q}{\lambda+1}} .
\end{aligned}
$$


Оценим каждый множитель. Умножим уравнение (1.1) на $u^{\vartheta}$ и, проинтегрировав по $\mathbb{R}^{N+M}$, получим

$\frac{d}{d t} \int_{\mathbb{R}^{N+M}} u^{1+\vartheta} d z+\int_{\mathbb{R}^{N+M}} u^{\vartheta-1}\left|D_{L} u\right|^{\lambda+1} d z+\int_{\mathbb{R}^{N+M}} a(\rho(z)) f(t)\left|D_{L} u^{\nu}\right|^{q} u^{\vartheta} d z=0$.

Откуда имеем

$$
H_{1} \leqslant-C \frac{d}{d \tau} \int_{\mathbb{R}^{N+M}} u^{1+\vartheta} d z .
$$

Для оценки второго множителя используем предложение 7.

$$
\begin{aligned}
H_{2} & =\int_{\mathbb{R}^{N+M}} a(\rho(z))^{\frac{\lambda+1}{\lambda+1-q}} f(t)^{\frac{\lambda+1}{\lambda+1-q}} u^{\frac{(\nu-1) q(\lambda+1)}{\lambda+1-q}-\frac{(\vartheta-1) q}{\lambda+1-q}-1+1} d z \\
& \leqslant \int_{\mathbb{R}^{N+M}} u(z, t) a\left(C\left\|u_{0}\right\|_{1}^{\frac{\lambda+1}{K}} t^{\frac{1}{K}}\right) f(t)^{\frac{\lambda+1}{\lambda+1-q}}\|u\|_{\infty, \mathbb{R}^{N+M}}^{\frac{H-\vartheta q}{\lambda+1}} d z \\
& =\|u(t)\|_{1, \mathbb{R}^{N+M}} a\left(C\left\|u_{0}\right\|_{1}^{\frac{\lambda+1}{K}} t^{\frac{1}{K}}\right) f(t)^{\frac{\lambda+1}{\lambda+1-q}}\left(C\left\|u_{0}\right\|_{1}^{\frac{\lambda+1}{K}} t^{-\frac{Q}{K}}\right)^{\frac{H-\vartheta q}{\lambda+1-q}} \\
& \leqslant C\left(t_{0},\left\|u_{0}\right\|_{1}\right)\|u(t)\|_{1, \mathbb{R}^{N+M}}
\end{aligned}
$$

Получаем

$$
\begin{aligned}
H_{1}^{\frac{q}{\lambda+1}} H_{2}^{\frac{\lambda+1-q}{\lambda+1}} & \leqslant C\left(-\frac{d}{d t} E_{\vartheta}(t)\right)^{\frac{q}{\lambda+1}}\left(C\|u(t)\|_{1, \mathbb{R}^{N+M}}\right)^{\frac{\lambda+1-q}{\lambda+1}} \\
& \leqslant C_{\sigma}\|u(t)\|_{1, \mathbb{R}^{N+M}}-\sigma \frac{d}{d t} E_{\vartheta}(t)
\end{aligned}
$$

$\sigma$ будет выбрана позднее. Имеем

$$
\frac{d}{d \tau}\|u(\tau)\|_{1, \mathbb{R}^{N+M}} \geqslant \sigma \frac{d}{d \tau} E_{\vartheta}(\tau)-C_{\sigma}\|u(\tau)\|_{1, \mathbb{R}^{N+M}} .
$$

Проинтегрируем полученное неравенство по интервалу $\left(t, t_{0}\right)$, где $t$ близко к $t_{0}$, $t<t_{0}$.

$$
\left\|u\left(t_{0}\right)\right\|_{1, \mathbb{R}^{N+M}}-\|u(t)\|_{1, \mathbb{R}^{N+M}} \geqslant \sigma E_{\vartheta}\left(t_{0}\right)-\sigma E_{\vartheta}(t)-C_{\sigma} \int_{t}^{t_{0}}\|u(\tau)\|_{1, \mathbb{R}^{N+M}} d \tau .
$$

Поскольку очевидно, что $\|u(\tau)\|_{1, \mathbb{R}^{N+M}}$ убывает, так как производная отрицательна (см. (5.2)), имеем

$$
\begin{aligned}
\|u(t)\|_{1, \mathbb{R}^{N+M}} & \leqslant \sigma E_{\vartheta}(t)+C_{\sigma}\left(t_{0}-t\right)\|u(t)\|_{1, \mathbb{R}^{N+M}} \\
& \leqslant \sigma C^{\vartheta}\|u(t)\|_{1, \mathbb{R}^{N+M}}+C_{\sigma}\left(t_{0}-t\right)\|u(t)\|_{1, \mathbb{R}^{N+M}} .
\end{aligned}
$$

Выберем $\sigma, \sigma C\left(t_{0},\left\|u_{0}\right\|, 1\right)^{\vartheta}<1$, и разделим неравенство на $\|u(t)\|_{1, \mathbb{R}^{N+M}}>0$. Получим $1 \leqslant \sigma C\left(t_{0},\left\|u_{0}\right\|, 1\right)^{\vartheta}+C_{\sigma}\left(t_{0}-t\right)$, при $t \rightarrow t_{0}$ приходим к противоречию. Лемма доказана.

Проинтегрируем уравнение (1.1) на множестве $\mathbb{R}^{N+M} \times\left(t_{1}, t\right)$. Аналогично доказанному в лемме 7.1, получим

$$
\left\|u\left(t_{1}\right)\right\|_{1, \mathbb{R}^{N+M}}-\|u(t)\|_{1, \mathbb{R}^{N+M}}=\int_{t_{1}}^{t} \int_{\mathbb{R}^{N+M}} a(\rho(z)) f(\tau)\left|D_{L} u^{\nu}\right|^{q} d z d \tau
$$




$$
\begin{aligned}
= & \int_{t_{1}}^{t} \int_{\mathbb{R}^{N+M}} a(\rho(z)) f(\tau)\left|\nu u^{\nu-1} D_{L} u\right|^{q} u^{\frac{(\vartheta-1) q}{\lambda+1}} u^{-\frac{(\vartheta-1) q}{\lambda+1}} d z d \tau \\
\leqslant & C\left(\int_{t_{1}}^{t} \int_{\mathbb{R}^{N+M}} u^{\vartheta-1}\left|D_{L} u\right|^{\lambda+1} d z d \tau\right)^{\frac{q}{\lambda+1}} \\
& \quad \times\left(\int_{t_{1}}^{t} \int_{\mathbb{R}^{N+M}} a(\rho(z))^{\frac{\lambda+1}{\lambda+1-q}} f(\tau)^{\frac{\lambda+1}{\lambda+1-q}}\left[u^{(\nu-1) q-\frac{(\vartheta-1) q}{\lambda+1}}\right]^{\frac{\lambda+1}{\lambda+1-q}} d z d \tau\right)^{\frac{\lambda+1-q}{\lambda+1}} \\
= & C P_{1}^{\frac{q}{\lambda+1}} P_{2}^{\frac{\lambda+1-q}{\lambda+1}} .
\end{aligned}
$$

Используя (7.1), имеем

$$
\begin{aligned}
P_{1} & =\int_{t_{1}}^{t} \int_{\mathbb{R}^{N+M}} u^{\vartheta-1}\left|D_{L} u\right|^{\lambda+1} d z d \tau \leqslant C\left\|u^{1+\vartheta}\left(t_{1}\right)\right\|_{1, \mathbb{R}^{N+M}} \\
& \leqslant C\left\|u\left(t_{1}\right)\right\|_{1, \mathbb{R}^{N+M}}\left\|u_{0}\right\|_{1}^{\frac{(\lambda+1) \vartheta}{K}} t_{1}^{-\frac{Q \vartheta}{K}} .
\end{aligned}
$$

Пусть выполняется оценка (1.8), тогда для достаточно больших $t$ справедлива оценка $Z(t) \leqslant C t^{\frac{1}{K}} \leqslant C \omega(t) t^{\frac{1}{K}}$. Используя предложение 8 и закон сохранения массы для суперзадачи без демпфирования, имеем

$$
\begin{aligned}
P_{2} & =\int_{t_{1}}^{t} \int_{\mathbb{R}^{N+M}} a(\rho(z))^{\frac{\lambda+1}{\lambda+1-q}} f(\tau)^{\frac{\lambda+1}{\lambda+1-q}} u^{\frac{(\nu-1) q(\lambda+1)}{\lambda+1-q}-\frac{(\vartheta-1) q}{\lambda+1-q}} d z d \tau \\
& \leqslant\left\|u\left(t_{1}\right)\right\|_{1, \mathbb{R}^{N+M}} \int_{t_{1}}^{t} C a\left(\omega(\tau) \tau^{\frac{1}{K}}\right)^{\frac{\lambda+1}{\lambda+1-q}} f(\tau)^{\frac{\lambda+1}{\lambda+1-q}}\|u(\tau)\|_{\infty, \mathbb{R}^{N+M}}^{\frac{H-\vartheta q}{\lambda+1}} d \tau \\
& \leqslant\left\|u\left(t_{1}\right)\right\|_{1, \mathbb{R}^{N+M}} \int_{t_{1}}^{t} C a\left(\omega(\tau) \tau^{\frac{1}{K}}\right)^{\frac{\lambda+1}{\lambda+1-q}} f(\tau)^{\frac{\lambda+1}{\lambda+1-q}} \tau^{-\frac{Q(H-\vartheta q)}{K(\lambda+1-q)}} d \tau .
\end{aligned}
$$

Используя равенство (4.5), получим

$$
a\left(\omega(\tau) \tau^{\frac{1}{K}}\right)^{\frac{\lambda+1}{\lambda+1-q}} f(\tau)^{\frac{\lambda+1}{\lambda+1-q}} \tau^{-\frac{Q(H-\vartheta q)}{K(\lambda+1-q)}}=\frac{\tau^{\frac{\vartheta q Q}{K(\lambda+1-q)}}}{\tau \omega^{\frac{H(\lambda+1)}{(\lambda-1)(\lambda+1-q)}}(\tau)} .
$$

Если $\vartheta$ выбрать достаточно маленьким, то

$$
\begin{aligned}
& a\left(\tau^{\frac{1}{K}}\right)^{\frac{\lambda+1}{\lambda+1-q}} f(\tau)^{\frac{\lambda+1}{\lambda+1-q}} \tau^{-\frac{Q(H-\vartheta q)}{K(\lambda+1-q)}} \\
& =\tau^{-1-\epsilon \frac{H(\lambda+1)}{(\lambda-1)(\lambda+1-q)}} \tau^{\frac{\vartheta q Q}{K(\lambda+1-q)}}=\tau^{-1-\left(\epsilon \frac{H(\lambda+1)}{(\lambda-1)(\lambda+1-q)}-\frac{\vartheta q Q}{K(\lambda+1-q)}\right)}, \\
& -\left(\epsilon \frac{H(\lambda+1)}{(\lambda-1)(\lambda+1-q)}-\frac{\vartheta q Q}{K(\lambda+1-q)}\right)<0, \\
& P_{2} \leqslant\left\|u\left(t_{1}\right)\right\|_{1, \mathbb{R}^{N+M}} C\left(\left\|u_{0}\right\|_{1}\right) t_{1}^{-\left(\epsilon \frac{H(\lambda+1)}{(\lambda-1)(\lambda+1-q)}-\frac{\vartheta q Q}{K(\lambda+1-q)}\right)}, \\
& \left\|u\left(t_{1}\right)\right\|_{1, \mathbb{R}^{N+M}} \leqslant\|u(t)\|_{1, \mathbb{R}^{N+M}}+C\left\|u\left(t_{1}\right)\right\|_{1, \mathbb{R}^{N+M}} t_{1}^{-\epsilon \frac{H}{\lambda-1}} .
\end{aligned}
$$

Отсюда, при достаточно больших $t_{1}$, имеем

$$
\left\|u\left(t_{1}\right)\right\|_{1, \mathbb{R}^{N+M}} \leqslant\|u(t)\|_{1, \mathbb{R}^{N+M}}+\frac{1}{2}\left\|u\left(t_{1}\right)\right\|_{1, \mathbb{R}^{N+M}} .
$$


Иначе говоря, мы доказали, что $\|u(t)\|_{1, \mathbb{R}^{N+M}} \geqslant \frac{1}{2}\left\|u\left(t_{1}\right)\right\|_{1, \mathbb{R}^{N+M}}, t>t_{1}$. По лемме 7.1

$$
\frac{1}{2}\left\|u\left(t_{1}\right)\right\|_{1, \mathbb{R}^{N+M}} \equiv C>0
$$

так как $t_{1}>0$. Теорема доказана.

\section{Список литературы}

[1] N. Garofalo, D.-M. Nhieu, "Isoperimetric and Sobolev inequalities for Carnot-Caratheodory spaces and the existence of minimal surfaces", Comm. Pure Appl. Math., 49:10 (1996), 1081-1144.

[2] I. Kombe, Hardy and Rellich type inequalities with remainders for Baouendi-Grushin vector fields, 2007, arXiv: 0704.1343.

[3] А.С. Калашников, "Некоторые вопросы качественной теории нелинейных вырождающихся параболических уравнений второго порядка”, УМH, 42:2 (1987), 135-176; англ. пер.: A.S. Kalashnikov, "Some problems of the qualitative theory of non-linear degenerate second-order parabolic equations", Russian Math. Surveys, 42:2 (1987), 169-222.

[4] В.В. Грушин, "Об одном классе гипоэллиптических операторов", Матем. сб., 83(125):3(11) (1970), 456-473; англ. пер.: V. V. Grušin, "On a class of hypoelliptic operators", Math. USSR-Sb., 12:3 (1970), 458-476.

[5] M. S. Baouendi, "Sur une classe d'opérateurs elliptiques dégénérés", Bull. Soc. Math. France, 95 (1967), 45-87.

[6] B. Franchi, E. Lanconelli, "Une métrique associée à une classe d'opérateurs elliptiques degeneres", Conference on linear partial and pseudodifferential operators (Torino, 1982), Rend. Sem. Mat. Univ. Politec. Torino, Università e Politecnico, Toronto, 1984, 105-114.

[7] B. Franchi, E. Lanconelli, "Hölder regularity theorem for a class of linear nonuniformly elliptic operators with measurable coefficients", Ann. Scuola Norm. Sup. Pisa Cl. Sci. (4), 10:4 (1983), 523-541.

[8] В.А. Маркашева, А.Ф. Тедеев, “Локальные и глобальные оценки решений задачи Коши для квазилинейных параболических уравнений с нелинейным оператором типа Баоуенди-Грушина", Матем. заметки, 85:3 (2009), 395-407; англ. пер.: V. A. Markasheva, A.F. Tedeev, "Local and global estimates of the solutions of the Cauchy problem for quasilinear parabolic equations with a nonlinear operator of Baouendi-Grushin type", Math. Notes, 85:3 (2009), 385-396.

[9] В. А. Маркашева, “Локальная гёльдеровость решений квазилинейных параболических уравнений с нелинейным оператором типа Баоуенди-Грушина, I", Тр. ИПММ, 16, ИПММ НАН Украины, Донецк, 2008, 124-135.

[10] В. А. Маркашева, “Локальная гёльдеровость решений квазилинейных параболических уравнений с нелинейным оператором типа Баоуенди-Грушина, II”, Tp. ИПММ, 17, ИПММ НАН Украины, Донецк, 2008, 128-143.

[11] S. Benachour, B. Roynette, P. Vallois, "Asymptotic estimates of solutions of $u_{t}-$ $\frac{1}{2} \Delta u=-|\nabla u|$ in $R_{+} \times R^{d}, d \geqslant 2 "$, J. Funct. Anal., 144:2 (1997), 301-324.

[12] Th. Gallay, Ph. Laurençot, "Asymptotic behavior for a viscous Hamilton-Jacobi equation with critical exponent", Indiana Univ. Math. J., 56:1 (2007), 459-479.

[13] M. Ben-Artzi, "Global existence and decay for a nonlinear parabolic equation", Nonlinear Anal., 19:8 (1992), 763-768.

[14] G. Barles, F. Da Lio, "On the generalized Dirichlet problem for viscous HamiltonJacobi equations", J. Math. Pures Appl. (9), 83:1 (2004), 53-75. 
[15] L. Amour, M. Ben-Artzi, "Global existence and decay for viscous Hamilton-Jacobi equations", Nonlinear Anal., 31:5-6 (1998), 621-628.

[16] S. Benachour, Ph. Laurençot, "Global solutions to viscous Hamilton-Jacobi equations with irregular initial data", Comm. Partial Differential Equations, 24:11-12 (1999), 1999-2021.

[17] M. Ben-Artzi, H. Koch, "Decay of mass for a semilinear parabolic equation", Comm. Partial Differential Equations, 24:5-6 (1999), 869-881.

[18] A. Dall'Aglio, D. Giachetti, S. Segura de León, "Global existence for parabolic problems involving the $p$-Laplacian and a critical gradient term", Indiana Univ. Math. J., 58:1 (2009), 1-48.

[19] Ph. Laurençot, J. Vázquez, "Localized non-diffusive asymptotic patterns for nonlinear parabolic equations with gradient absorption", J. Dynam. Differential Equations, 19:4 (2007), 985-1005.

[20] S. Snoussi, S. Tayachi, "Large time behavior of solutions for parabolic equations with nonlinear gradient terms", Hokkaido Math. J., 36:2 (2007), 311-344.

[21] J.-Ph. Bartier, Ph. Laurençot, "Gradient estimates for a degenerate parabolic equation with gradient absorption and applications", J. Funct. Anal., 254:3 (2008), 851-878.

[22] Ch. Stinner, "Convergence to steady states in a viscous Hamilton-Jacobi equation with degenerate diffusion", J. Differential Equations, 248:2 (2010), 209-228.

[23] D. Andreucci, A. F. Tedeev, M. Ughi, "The Cauchy problem for degenerate parabolic equations with source and damping", Укр. матем. журн., 1:1 (2004), 1-19; англ. пер.: D. Andreucci, A.F. Tedeev, M. Ughi, "The Cauchy problem for degenerate parabolic equations with source and damping", Ukr. Math. Bull., 1:1 (2004), 1-23.

[24] А.Ф. Тедеев, "Начально-краевые задачи для квазилинейных вырождающихся параболических уравнений с демпфированием. Задача Неймана", Укр. матем. журн., 58:2 (2006), 272-282; англ. пер.: А. F. Tedeev, "Initial-boundary value problems for quasilinear degenerate parabolic equations with damping. The Neumann problem", Ukrainian Math. J., 58:2 (2006), 304-317.

[25] D. Andreucci, A.F. Tedeev, "Finite speed of propagation for the thin-film equation and other higher-order parabolic equations with general nonlinearity", Interfaces Free Bound., 3:3 (2001), 233-264.

[26] D. Andreucci, A.F. Tedeev, "Sharp estimates and finite speed of propagation for a Neumann problem in domains narrowing at infinity", Adv. Differential Equations, 5:7-9 (2000), 833-860.

[27] D. Andreucci, A. F. Tedeev, "A Fujita type result for a degenerate Neumann problem in domains with noncompact boundary", J. Math. Anal. Appl., 231:2 (1999), 543-567.

[28] D.S. Jerison, "The Dirichlet problem for the Kohn Laplacian on the Heisenberg group. II", J. Funct. Anal., 43:2 (1981), 224-257.

[29] S. K. Vodopyanov, "Foundations of the theory of mappings with bounded distortion on Carnot groups", The interaction of analysis and geometry, Contemp. Math., 424, Amer. Math. Soc, Providence, RI, 2007, 303-344.

[30] Е. А. Плотникова, "Интегральные представления и обобщенное неравенство Пуанкаре на группах Карно", Сиб. матем. журн., 49:2 (2008), 420-436; англ. пер.: E. A. Plotnikova, "Integral representations and the generalized Poincare inequality on Carnot groups", Siberian Math. J., 49:2 (2008), 339-352.

[31] О. А. Ладыженская, В. А. Солонников, Н. Н. Уральцева, Линейные и квазилинейные уравнения параболического типа, Наука, М., 1967; англ. пер.: О. А. Ladyzhenskaya, V.A. Solonnikov, N. N. Ural'tseva, Linear and quasi-linear equations of parabolic type, Transl. Math. Monogr., 23, Amer. Math. Soc., Providence, RI, 1968.

[32] E. DiBenedetto, Degenerate parabolic equations, Springer-Verlag, New York, 1993. 
[33] Ж.-Л. Лионс, Некоторые методы решения нелинейных краевых задач, Мир, М., 1972; пер. с фр.: J.-L. Lions, Quelques méthodes de résolution des problèmes aux limites non linéaires, Dunod, de Gruyter, Paris, 1969.

[34] D. Andreucci, "Degenerate parabolic equations with initial data measures", Trans. Amer. Math. Soc., 349:10 (1997), 3911-3923.

[35] H. Shang, F. Li, "On the Cauchy problem for the evolution $p$-Laplacian equations with gradient term and source and measures as initial data", Nonlinear Anal., 72:7-8 (2010), 3396-3411.

В. А. Маркашева (V. А. Markasheva)

Институт прикладной математики и механики

НАН Украины, г. Донецк

E-mail: w9071981@yandex.ru

\section{А. Ф. Тедеев (А. F. Tedeev)}

Институт прикладной математики и механики

НАН Украины, г. Донецк

E-mail: tedeev@iamm.ac.donetsk.ua
Поступила в редакцию 26.05 .2010 и 26.08 .2011 